\title{
1 Emergence of an adaptive epigenetic cell state in human bladder
}

\section{2 urothelial carcinoma evolution}

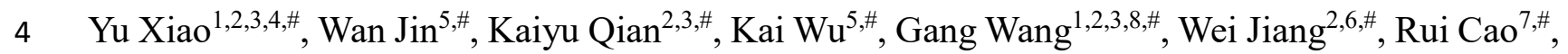

5 Lingao $\mathrm{Ju}^{2,3,8, \#}$, Yan Zhao ${ }^{9,10, \# \text {, Hang Zheng }}{ }^{1, \#}$, Tongzu Liu ${ }^{1}$, Luyuan $\mathrm{Chang}^{5}$, Zilin Xu ${ }^{2,3,8}$, Ting

6 Wang $^{1}$, Jun Luo ${ }^{11}$, Liuying Shan ${ }^{5}$, Fang Yu ${ }^{11}$, Xintong Chen ${ }^{5}$, Dongmei Liu ${ }^{5}$, Hong Cao ${ }^{11}$,

7 Zhonghua Yang ${ }^{1}$, Sheng $\mathrm{Li}^{2,3,4,8}$, Hongjie Shi ${ }^{2,3}$, Zhongqiang Guo ${ }^{1}$, Yan Gong ${ }^{2,3,8}$, Nan Liu ${ }^{2,3}$,

8 Shenjuan $\mathrm{Li}^{2,3}$, Yejinpeng Wang ${ }^{1}$, Xinyue $\mathrm{Cao}^{2,3}$, Wenjun Ding ${ }^{2,3}$, Wei Zhou ${ }^{12,13,14}$, Diansheng

$9 \mathrm{Cui}^{15}$, Ye Tian${ }^{7}$, Chundong $\mathrm{Ji}^{16}$, Yongwen $\mathrm{Luo}^{1}$, Xin Hong ${ }^{17}$, Haoli Ma ${ }^{2,3,8}$, Fangjin Chen ${ }^{18,19}$,

Minsheng Peng ${ }^{20,21}$, Yi Zhang ${ }^{5,19}$, Xinghuan Wang ${ }^{1,2,3,4,6,8, *}$

\section{Affiliations:}

${ }^{1}$ Department of Urology, Zhongnan Hospital of Wuhan University, Wuhan, China.

$14{ }^{2}$ Department of Biological Repositories, Zhongnan Hospital of Wuhan University, Wuhan, China.

$15{ }^{3}$ Human Genetic Resource Preservation Center of Hubei Province, Wuhan, China.

$16{ }^{4}$ Wuhan Research Center for Infectious Diseases and Cancer, Chinese Academy of Medical

17 Sciences, Wuhan, China.

$18 \quad{ }^{5}$ Euler Technology, ZGC Life Sciences Park, Beijing, China.

$19{ }^{6}$ Medical Research Institute, Wuhan University, Wuhan, China.

$20{ }^{7}$ Department of Urology, Beijing Friendship Hospital, Capital Medical University, Beijing, China.

$21{ }^{8}$ Cancer Precision Diagnosis and Treatment and Translational Medicine Hubei Engineering

22 Research Center, Zhongnan Hospital of Wuhan University, Wuhan, China.

$23{ }^{9}$ Emergency Center, Zhongnan Hospital of Wuhan University, Wuhan, China. 
${ }^{10}$ Hubei Clinical Research Center for Emergency and Resuscitation, Zhongnan Hospital of Wuhan

25 University, Wuhan, China.

$26{ }^{11}$ Department of Pathology, Zhongnan Hospital of Wuhan University, Wuhan, China.

$27 \quad{ }^{12}$ Institute of Hepatobiliary Diseases of Wuhan University, Wuhan, China.

$28 \quad{ }^{13}$ Transplant Center of Wuhan University, Wuhan, China.

$29 \quad{ }^{14}$ Hubei Key Laboratory of Medical Technology on Transplantation, Wuhan, China.

$30 \quad{ }^{15}$ Department of Urology, Hubei Cancer Hospital, Wuhan, China.

$31{ }^{16}$ Department of Urology, The Affiliated Hospital of Panzhihua University, Panzhihua, China.

$32 \quad{ }^{17}$ Department of Urology, Peking University International Hospital, Beijing, China.

${ }^{18}$ Center for Quantitative Biology, School of Life Sciences, Peking University, Beijing, China.

$34 \quad{ }^{19}$ Center for Life Sciences, Peking University, Beijing, China.

$35{ }^{20}$ State Key Laboratory of Genetic Resources and Evolution, Kunming Institute of Zoology,

36 Chinese Academy of Sciences, Kunming, China.

$37 \quad{ }^{21}$ University of Academy of Sciences, Kunming College of Life Science, Kunming, China.

$38 \quad$ \#These authors contributed equally to this work.

40 "Corresponding authors: Dr. Xinghuan Wang, Email: wangxinghuan@whu.edu.cn, Tel.: +86-27-

$41 \quad 6781-3104$. 


\section{Abstract}

43 Intratumor heterogeneity (ITH) of bladder cancer (BLCA) facilitates therapy resistance and 44 immune evasion to affect clinical prognosis directly. However, the molecular and cellular mechanism generating ITH in BLCA remains elusive. Here we show that a TM4SF1-positive cancer subpopulation (TPCS) drives ITH diversification in BLCA. By extensive profiling of the epigenome and transcriptome of BLCA from 79 donors across all stages, we elucidated the evolution trajectories of luminal and basal BLCA. TPCS emerges from the basal trajectory and shows extensive transcriptional plasticity with a distinct epigenomic landscape. Clinically, TPCS were enriched in advanced stage patients and associated with poor prognosis. Our results showed how cancer adapts to its environment by adopting a stem cell-like epigenomic landscape.

\section{Introduction}

54 Bladder urothelial carcinoma (BLCA), which causes 212,500 deaths worldwide annually ${ }^{1}$, is a biologically diverse ensemble. The phenotypic diversity, in other words, intratumor heterogeneity (ITH) of BLCA determined their biological behaviour. ITH correlates with clinical stages, mutation burdens, and DNA copy number changes ${ }^{2}$ and less favourable survival outcome in both $\mathrm{BLCA}^{3}$ and other cancer types ${ }^{4-7}$. Whilst previous studies in other cancer types suggested that epigenetic plasticity $^{8-13}$ and genomic mutation ${ }^{9,10,14}$ lead to ITH, the exact molecular mechanism generating ITH in BLCA remains elusive. Clinically, BLCA are classified into two distinct categories: the less malignant non-muscle-invasive BLCA (NMIBC), including Ta, T1 and/or CIS (Tis) stages, and muscle-invasive BLCA (MIBC) with rapid growth rate and metastasis potential ${ }^{14-}$ 
and luminal-like phenotypes might co-exist in the same tumour or different urinary tract tumours of a single individual ${ }^{9,23}$ suggests interconvertibility between the classes. Therefore, the cellularorigin, genetic diversity, and epigenetic heterogeneity might all contribute to ITH in BLCA.

\section{Results}

\section{Single cell landscape of normal bladder and BLCA}

To characterize BLCA evolution from the single cell (sc) level (Supplementary Fig. 1), we performed scRNA and scATAC sequencing on 56 tissues from 26 donors, including 23 BLCA patients and 3 Donations after Cardiac Death (DCD) (Supplementary Table 1-4). Also, scRNA data of 11 BLCA tissues from the public domain ${ }^{24}$ was included. After stringent quality control ${ }^{25-27}$, 133,953 cells remained, including epithelia, endothelia, fibroblasts, myeloid cells, $\mathrm{CD}^{+}{ }^{\mathrm{T}}-$ lymphocytes, B-lymphocytes, and NK cells (Fig. 1, Supplementary Table 3-6). These cells were derived from different donors (Fig. 1C) and different tissue types (Fig. 1D).

Epithelial cells dominate the population in cancer (Fig. 1E). Driven by donor-specific copy number variation $(\mathrm{CNV})$, urothelium epithelia originated cancer cells segregated into donor-specific clusters, whilst CNV-free cells from tumour and normal urothelial tissues tended to cluster together (Fig. 1E-F). Luminal-like and basal-like cancer cells could be identified by the expression of GATA3/FGFR3 or KRT5/KRT6A/KRT7, respectively (Fig. 1E and Supplementary Fig. 2). Two populations of cancer cells are marked with robust proliferating gene expression: a TM4SF1positive cancer subpopulation (TPCS), and a TM4SF1-negative, fast proliferating cell population (FPC) (Fig. 1E-G and 3H-I). Both TPCS and FPC highly expressed cell-cycle related genes such 
as $H 2 A Z 1$ and $H 2 A Z 2$, whilst TPCS additionally overexpressed TM4SF1, PI3, and a set of keratins (KRT7/KRT6A/KRT6B) (Fig. 3I and Supplementary Fig. 2).

We further characterized 1,163 normal epithelial cells from healthy organ donors in detail. These cells were classified into basal (Bs1/2/3), intermediate ( $\operatorname{Im} 1 / 2 / 3)$, umbrella (Um) by the expression of $S H H, K R T 5$ and uroplakins ${ }^{28}$ and a small number of urothelium stem/progenitor cell (BsP) (Fig. 2A-B). BsP is highly proliferative (Fig. 2C) and overexpressed TM4SF1 (Fig. 3I). Basal cell lineage is clearly separated from the superficial cells not only by conventional markers KRT5/UPK2/UPK3A/UPK3B but also by HES1 and VEGFA expression (Fig. 2D). Bs2/3 lineage are distinct from BsP/Bs1 by that they overexpressed PCSK5 and SGK1 and have no HBEGF expression (Fig. 2D), suggesting a functional heterogeneity between the basal cells.

The bifurcation of HES1/HES4 expression levels in basal and superficial (intermediate/umbrella) cells suggests that Notch signalling might contribute to urothelium cell lineage specification (Fig. 2E). In healthy bladder tissue, the expression of Notch ligand $J A G 1$ was high in endothelial cells and fibroblasts compared to the epithelial cells. Meanwhile, basal cells expressed more NOTCH3 compared to superficial cells (Fig. 2E). Cell-cell signalling between the urothelium epithelial cells suggested that whilst only Bs1 provides EGF signal, and only intermediate cells provide TGF $\beta$ and BMP signal to other cells, Notch signalling is mutual between basal and superficial lineage cells (Fig. 2F). In concordance to previous reports that fibroblasts communicate with BLCA ${ }^{24,29}$, these results suggest that basal/superficial cell lineage bifurcation might be driven by Notch signalling. The physical proximity of basal cells to the underlying endothelial and fibroblast cells, which provides JAG1/DLL1 ligand, might determine their fate (Fig. 2G). 


\section{Transformation of bladder cancer cells from normal epithelium}

112 Strong transcriptional heterogeneity of BLCA epithelial cells masked underlying common

113 molecular determinants of their evolution. To study BLCA evolution from normal urothelium

114 epithelial cells, genome-wide DNA methylation and single cell chromatin accessibility (scATAC)

115 sequencing were performed (Methods). After quality control, 376,740 cells were obtained in 116 scATAC data $^{30}$, including 107,380 epithelial cells (Supplementary Table 4 and 6-7). To avoid

117 influence from CNV, we clustered the non-immune cells only with variable features from copy118 number stable regions (Methods). To this end, 15 clusters of single cells with distinct chromatin 119 accessibility landscapes on differentially methylated regions were identified and termed as

120 'epigenotype' (Supplementary Fig. 3B, Supplementary Table 2 and 9). The distribution of 121 epigenotypes in each tumour was associated with BLCA clinical stages (Supplementary Fig. 3C).

122 Epigenotype Cluster1-4 were stromal cells, whilst Cluster5-15 were epithelial cells 123 (Supplementary Fig. 3B and D). By integrating scRNA-seq data with scATAC, RNA counterparts 124 were matched with epigenotypes (RNA clusters in Supplementary Table 6): normal urothelium 125 basal cells correspond to Cluster14; RNA-determined TPCS cells correspond to Cluster5-7; basal126 like cancer cells correspond to Cluster8-13 and luminal-like cancer cells correspond to Cluster15. 127 Similar to the scRNA-inferred developmental trajectory of cancer cells, pseudotime tracing 128 showed that all cancer epithelial cells developed from normal-like Cluster14 cells in scATAC 129 (Methods and Supplementary Fig. 3A).

131 It is well known that oncogenesis is accompanied by a drastic change in DNA methylation 132 landscape. We found that genomic regions that are hypermethylated in BLCA (hyperDMR) were 
133

134

135

136

137

138

139

140

141

142

143

144

145

146

147

148

149

150

151

152

153

154 generally open in stromal cells and remained closed in all epithelial cells regardless of their malignancy states; in contrast, genomic regions that are hypomethylated in BLCA (hypoDMR) is only open in cancer but not normal epithelial cells (Fig. 3A-C and Supplementary Table 2). As DNA methylation is usually accompanied by an inaccessible chromatin state, these results suggest that DNA methylation levels on most hyperDMR are invariable during oncogenesis and the apparent hypermethylation of these regions in tumour tissue is a mere result of cancer clonal expansion. In contrast, active DNA demethylation occurs on hypoDMR accompanied oncogenesis. Hence, hyperDMR can be used as a tool for lineage tracing.

We identified a core subset of hyperDMR which can distinguish MIBC and NMIBC by unsupervised hierarchical clustering. NMIBC Ta and T1-T4 tumours could be classified by DNA methylation levels on different groups of core hyperDMR (Fig. 3D), suggesting they might have distinct cell-of-origins. By correlating gene expression and DMR methylation, we find that NMIBC-specific-hypermethylated regions are associated with basal-specific, superficial-low genes (GPX4/PRKCZ/CD44), suggesting that NMIBC originates from superficial cell lineage. On the contrary, MIBC-specific-hypermethylated regions are associated with genes with lower expression in basal cells. Interestingly, a subgroup of T3/T4 MIBC is hypermethylated in all core hyperDMR and correlates with $\mathrm{BsP}$ origin by associated gene expression, indicating BsP originated cancer cells are most malignant (Fig. 3D-E). Taken together, these results suggest a heterogeneous origin of BLCA: NMIBC originates from superficial urothelium cells and MIBC develop from basal cells. Finally, the developmental trajectory of MIBC evolution (Fig. 3F) showed that TPSC and FPC are both bifurcated from basal cancer cells (Ca) (Fig. 3G). Whilst most 
differential expressed genes in FPC are proliferation-related, downregulated Notch signalling and upregulated keratin genes expression in TPCS suggested a phenotype shift (Fig. 3H-I).

Cell-cell signalling analysis showed that FPC specifically received VEGF signals from $\mathrm{Ca}$, and TPCS specifically received BMP signals (BMP2/4 and GDF5) from Ca (Fig. 4A), suggesting BMP signalling might be the extrinsic driver for TPCS genesis. Non-negative matrix factorization $(\mathrm{NMF})^{31,32}$ identified three independent gene sets (metagenes) NMF6, NMF11 and NMF16, which are highly expressed in TPCS compared to Ca and FPC (Fig. 4B-C). Promoters of these metagenes were enriched with transcription factor binding sites (TFBS) ${ }^{33,34}$ of SMAD4, ASCL2, NFIB, MEF2C, GLI1, BACH1, BACH2 and BATF (Supplementary Fig. 4-5), suggesting that they are co-regulated (Supplementary Table 7). While NMF16 genes are under the control of NFIB, GLI1/3 and MEF2C, NMF6 and NMF11 genes are directly controlled by BMP via SMAD4 and Notch via ASCL2 (Fig. 4D). As ASCL1/2 expression is difficult to be assessed in scRNA-seq, we used scATAC data to infer their transcriptional activity. In concordance with the NMF result, ASCL1/2 and SMAD4 TF activities are increased in TPCS without apparent transcriptional upregulation (Fig. 4E).

\section{Emergence of a transcriptional plastic cancer subpopulation by epigenetic reprogramming}

We investigated genome-wide distribution of the MIBC master transcription factors FOXA1 ${ }^{35}$ and AR as well as $\mathrm{H} 3 \mathrm{~K} 4 \mathrm{me} 3, \mathrm{H} 3 \mathrm{~K} 27 \mathrm{ac}$ and $\mathrm{H} 3 \mathrm{~K} 27 \mathrm{me} 3$ on BLCA cell lines (Fig. 5A-B). Two cell lines were chosen for Cut\&Tag sequencing analysis: RT4 cell line with TACC3-FGFR3 representing NMIBC (Luminal, L), and 5637 cell line representing MIBC (Basal, B) (Fig. 5A-B 
177

178

179

180

181

182

183

184

185

186

187

188

189

190

191

192

193

194

195

196

197

198

199

and Supplementary Table 8). Of particular note, as 5637 expressed a high level of $K R T 6 A / B$, we consider it is a basal-like cell line that mimics TPCS.

Histone modifications and transcription factor binding profiles grouped MIBC-specific hypoDMR into 10 clusters (Methods). MIBC-hypoDMR group3 (hypoC3) lost AR binding as well as $\mathrm{H} 3 \mathrm{~K} 27 \mathrm{ac}$ modification, and the chromatin accessibility on hypoC3 is increased in basal cancer cells compared to luminal cancer cells, and further increased in TPCS (Fig. 5C). MIBC-hypoDMR group7 (hypoC7) is also identified as enhancers that are bound by FOXA1 and lost H3K27ac in basal cells (Fig. 5D). This group of enhancer controls several basal-low genes such as GPX4 and YWHAZ, as well as HES4. Chromatin accessibility on hypoC7 and hypoC3 is highest in TPCS (Fig. 5C-D). On the other hand, MIBC-hyperDMR group8 (hyperC8) is a group of FOXA1-bound, H3K27ac/H3K4me3-high enhancers (Fig. 5B), which controls the TPCS-specific transcription factor JDP2 and TFAP2C; and MIBC-hyperDMR group6 (hyperC6) is a group of promoters that is only active in basal cancer cells that control lineage specification genes such as HOXA3/HOXA6/DMRTA2 (Fig. 5B). Furthermore, additional loci that were differentially methylated in MIBC are shadowed by H3K27me3 in basal cells only, suggesting PRC2-dependent regulation. In concordance with this result, we observed YY1 binding activity is significantly downregulated in TPCS (Supplementary Fig. 4).

Such epigenomic landscape shift has an immediate effect on gene expression. In TPCS-enriched T3 and T4a samples, DNA demethylation around a distal FOXA1 and CTCF-binding loci 3' to HES4 lead to loss of long-range chromatin interaction, loss of enhancer activity, and gain of repressive H3K27me3 mark around HES4 locus (Fig. 5E-F). Similarly, differential FOXA1 
binding and DNA methylation led to increased interaction between FOXA1-bound active enhancer to TFAP2C promoter in TPCS (Fig. 5G-H). Together, these results suggest that histone modification and DNA methylation changes occur concomitantly with differential AR/FOXA1 binding sites, and might control TPCS-specific transcription programs.

As clinical stage advanced, the predominant population of epigenotype shifted towards TPCS (Fig. 6A). Matching TPCS cells to their CNV profile and integrated RNA data showed that dormant normal cells, proliferating normal cells, and fast-proliferating cancer cells have distinct epigenotype profiles. Whilst the majority of TPCS cells from scRNA clustered to one single CNV type, possibly due to sampling bias, epigenotype of these TPCS cells could correspond to multiple different clusters of scRNA cells (Fig. 6B). In general, the diversity of RNA expression matched to TPCS epigenotype is higher than those matched to other basal cancer cell epigenotypes (Fig. 6C). Orthogonal validation using gene expression diversity analysis (CytoTRACE) showed that FPC and TPCS are likely to be the most undifferentiated, stem-like cells compared to basal and luminal cancer cells (Fig. 6D). Overall, these results suggest that the epigenomic reprogramming in TPCS created a fluid epigenotype that supports multiple transcriptionally diverse phenotypes. In other words, TPCS epigenotype creates ITH without further genetic mutations.

\section{TPCS heterogeneity supports immune evasion and chemotherapy resistance}

ITH created by TPCS might be beneficial for the survival of cancer cells. On one hand, ITH might facilitate immune evasion by shifting neoantigen expression or directly upregulating immune suppression molecules. On the other hand, ITH might facilitate drug resistance by creating a pool of diverse cancer cells that responses differentially to therapy. We studied the immunoediting in 
tumour tissues by characterizing intratumoral T-lymphocyte population and neoantigen expression in cancer cells. T-lymphocyte clonal expansion status was defined by TCR clone sequence prevalence across tissues. Clonal expansion of T-lymphocyte was absent in healthy or NMIBC Ta BLCA donors but present in T1-T4 donors, and prevalence of clonally-expanded T-lymphocyte and diversity of TCR clone correlates with T-stage, suggesting that whilst bystander (bs) Tlymphocyte infiltration is prevalent in BLCA, they do not mask real tumour-reactive (tr) $\mathrm{T}$ lymphocyte responses, as clonal expansion is still robust (Fig. 7A-B).

For $\mathrm{CD}^{+} \mathrm{T}$-cell, clonal expansion is more prevalent in the mature T-cell population (bsTh2/17, trTh1, cTreg, eTreg, trTreg) (Fig. 7C and Supplementary Fig. 6). Among these CD4 ${ }^{+}$T-cell, globally-expanded population (bsTh2/17, eTreg) do not express ITGAE(CD103) and ENTPD1(CD39) (Fig. 7D and Supplementary Fig. 6). On the contrary, locally-expanded trTh1 and trTreg overexpress ITGAE/ENTPD1 in addition to RGS2/IRF1/TNFRSF4 (Fig. 7D and Supplementary Fig. 6). In the light of previous reports ${ }^{36}$ and considering the local TCR clone expansion, these results suggested that CD103/CD39 combination might serve as a general marker for tumour-reactiveness ${ }^{37}$. Cell-cell signalling analysis suggests that trTreg contributed a majority of PD-L1 ligand to other T cells in the tumour environment (Fig. 7E). The prevalence of trTreg increased in T3 whilst trTh1 decreased over clinical stage progression (Fig. 7F-G). For cytotoxic $\mathrm{CD}^{+} \mathrm{T}$ cells in tumour environment, we found that the globally-expanded cells include the immature, non-PD-1-expressing $\mathrm{T}_{\mathrm{emra}}(\mathrm{KLRG} 1), \mathrm{T}_{\mathrm{emr}}(\mathrm{PRF} 1), \mathrm{T}_{\mathrm{emra}}(\mathrm{XCL})$ and $\mathrm{PD}-1$ expressing (non-locally-expanded) act $\mathrm{T}_{\text {eff }}(\mathrm{GZMK})$ (Fig. 7H-I and Supplementary Fig. 7). Clonal expansion together with PDCD1 expression classified four clusters of $\mathrm{T}_{\text {eff: }}$ the $Z E B 2^{+} / T O P 2 A^{+}$proliferating

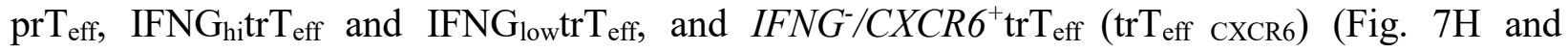


Supplementary Fig. 7). These tr $\mathrm{T}_{\text {eff }}$ were more prevalent in cancer tissues, with $\operatorname{tr} \mathrm{T}_{\text {eff }}$ CXCR6 is the

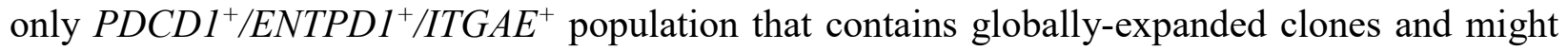
support strong antitumor activity ${ }^{38}$ (Fig. 7H, J-L and Supplementary Fig. 7).

The above results suggest BLCA tumour microenvironment is not completely immunosuppressive and continues to attract and train new naïve T cells to tumour-reactive controllers. Hence, BLCA might evade immunosurveillance via alternative mechanisms. We analyzed MHC-related molecule expression and mutation-carrying ('neoantigen') transcript expression in cancer cell scRNA data (Supplementary Table 9). Whilst MHC-related molecule expression is higher in TPCS than basal cancer cells, marginally significant fewer TPCS expressed mutation-carrying transcript than basal cancer cells (Fig. 7M). In basal cancer cells, mutation-carrying transcript expression level reversely correlates with the clinical stage. In contrast, TPCS expressing mutation-carrying transcripts remained stable (Fig. $7 \mathrm{~N}$ ). These results suggest that TPCS switch between mutationhigh and mutation-free states to evade immunosurveillance. Finally, the gene-set activity of TPCS marker metagene NMF11 component was used to quantify TPCS prevalence in BLCA from IMvigor210 dataset. Compared to immune-desert tumours, inflamed tumours are more likely to contain more TPCS (Fig. 7O). In the group of patients who received immunocheckpoint inhibitor (ICI) treatment, patients with high TPCS marker gene expression have significantly poorer prognosis (Fig. 7P), suggesting that TPCS contributed to ICI resistance.

A subgroup of patients in our cohort received adjuvant intravesical gemcitabine instillation. Whilst epigenotype-related phenotype diversity does not change between treatment-naïve and postchemotherapy patients for TPCS (Fig. 8A), the prevalence of TPCS but no other basal cancer cells 
269 is significantly increased after chemotherapy in different clinical stages (Fig. 8B-D). Cell cycle

270 score revealed that after gemcitabine treatment, the majority of basal-like and luminal-like cancer

271 cells tend to be arrested in G1 and do not enter S or G2/M phases (Fig. 8E). Additionally, FPC tend

272 to be arrested in G2/M, and TPCS become more active as the prevalence of both S and G2/M-

273 phase cells increased (Fig. 8E). In TCGA BLCA cohort, where patients were mainly treated with

274 chemotherapy, high TPCS marker gene expression significantly correlates with poor prognosis

275 (Fig. 8F), suggesting that TPCS contributed to chemotherapy resistance.

276 


\section{Discussion}

278 BLCA is a complicated and heterogeneous disease. The evolution of BLCA from normal urothelium to generate such heterogeneity remains unclear ${ }^{20}$. Particularly, no direct evidence was made from the molecular level to clarify the origins of basal/luminal types of human BLCA.

281 Through DNA methylation based lineage tracing and scATAC-seq, our results revealed a comprehensive picture of BLCA evolution. NMIBC and MIBC originate from superficial intermediate/umbrella or basal cells of the urothelium, respectively. Cell-of-origin of BLCA corresponds to their clinical behaviour, with BLCA originating directly from BsP being most malignant. In late stage, a progenitor-like, TM4SF1-positive cancer cell subpopulation emerge from the MIBC track. TPCS is induced by epigenetic reprogramming, and contributes to ITH by supporting highly plastic transcriptional phenotypes (Supplementary Fig. 8).

TM4SF1, a member of the tetraspanin family, has been identified as a tumour-specific antigen, 290 promoting proliferation, invasion, epithelial-mesenchymal transition (EMT) and chemoresistance $^{39-41}$. Expression of TM4SF1 marks stem cell in mesenchymal tissue ${ }^{42}$ and lung ${ }^{43}$. Here,

292 we identified that TM4SF1 also marks human bladder urothelium progenitor cell. Additionally, 293 TM4SF1-positive cancer cell subpopulation is also highly proliferative and transcriptionally 294 plastic. TPCS is phenotypically similar to hyper-plastic cancer cells reported in other cancer 295 types ${ }^{7,8,44,45}$. In our dataset, all metastatic, squamous differentiation BLCA-isolated cells are TPCS. 296 TPCS expresses lower HES1, HES4 and Notch pathway downstream targets, possessing the 297 characters of cancer stem-like cells (CSCs). Consistent with the notion that the phenotype of CSCs 298 is often regulated by epigenetic modifications ${ }^{44}$, our findings suggested that epigenomic 299 reprogramming in TPCS results in a variety of distinct transcriptional phenotypes. These data 
300 suggested that during BLCA progression, the adaptation of a progenitor/stem-cell like fate 301 occurred and contributed to ITH in BLCA. The emergence of TPCS increased phenotypic diversity 302 in tumours and facilitated immune evasion and chemotherapy resistance (Supplementary Fig. 8).

303 Our study provides not only insights into BLCA biology but also novel clinical targets for BLCA 304 therapy. 
306

307

308

309

310

311

312

313

314

315

316

317

318

319

320

321

322

323

324

325

326

327

328

\section{References}

1. Sung, H. et al. Global Cancer Statistics 2020: GLOBOCAN Estimates of Incidence and Mortality Worldwide for 36 Cancers in 185 Countries. CA Cancer J Clin 71, 209-249 (2021).

2. Cazier, J.B. et al. Whole-genome sequencing of bladder cancers reveals somatic CDKN1A mutations and clinicopathological associations with mutation burden. Nat Commun 5, 3756 (2014).

3. Liu, D. et al. Mutational patterns in chemotherapy resistant muscle-invasive bladder cancer. Nat Commun 8, 2193 (2017).

4. Dentro, S.C. et al. Characterizing genetic intra-tumor heterogeneity across 2,658 human cancer genomes. Cell 184, 2239-2254 e39 (2021).

5. Stewart, C.A. et al. Single-cell analyses reveal increased intratumoral heterogeneity after the onset of therapy resistance in small-cell lung cancer. Nat Cancer 1, 423-436 (2020).

6. Jamal-Hanjani, M. et al. Tracking the Evolution of Non-Small-Cell Lung Cancer. N Engl JMed 376, 2109-2121 (2017).

7. Patel, A.P. et al. Single-cell RNA-seq highlights intratumoral heterogeneity in primary glioblastoma. Science 344, 1396-401 (2014).

8. Marjanovic, N.D. et al. Emergence of a High-Plasticity Cell State during Lung Cancer Evolution. Cancer Cell 38, 229-246 e13 (2020).

9. Meeks, J.J. et al. Genomic heterogeneity in bladder cancer: challenges and possible solutions to improve outcomes. Nat Rev Urol 17, 259-270 (2020).

10. Faltas, B.M. et al. Clonal evolution of chemotherapy-resistant urothelial carcinoma. Nat Genet 48, 1490-1499 (2016). 
329 11. Hao, J.J. et al. Spatial intratumoral heterogeneity and temporal clonal evolution in esophageal squamous cell carcinoma. Nat Genet 48, 1500-1507 (2016).

12. Torres, C.M. et al. The linker histone H1.0 generates epigenetic and functional intratumor heterogeneity. Science 353(2016).

13. de Bruin, E.C. et al. Spatial and temporal diversity in genomic instability processes

14. Babjuk, M. et al. European Association of Urology Guidelines on Non-muscle-invasive (2019).

15. Witjes, J.A. et al. European Association of Urology Guidelines on Muscle-invasive and Metastatic Bladder Cancer: Summary of the 2020 Guidelines. Eur Urol 79, 82-104 (2021).

16. Chang, S.S. et al. Treatment of Non-Metastatic Muscle-Invasive Bladder Cancer: AUA/ASCO/ASTRO/SUO Guideline. J Urol 198, 552-559 (2017).

17. Chang, S.S. et al. Diagnosis and Treatment of Non-Muscle Invasive Bladder Cancer: AUA/SUO Guideline. J Urol 196, 1021-9 (2016).

18. Zhou, B. et al. Polymorphisms and plasma levels of IL-27: impact on genetic

19. van den Bosch, S. \& Alfred Witjes, J. Long-term cancer-specific survival in patients with high-risk, non-muscle-invasive bladder cancer and tumour progression: a systematic review. Eur Urol 60, 493-500 (2011). Cell Biol 16, 982-91, 1-5 (2014). 
352 21. Shin, K. et al. Cellular origin of bladder neoplasia and tissue dynamics of its progression to invasive carcinoma. Nat Cell Biol 16, 469-78 (2014).

354 22. Guo, C.C. et al. Assessment of Luminal and Basal Phenotypes in Bladder Cancer. Sci Rep $355 \quad \mathbf{1 0}, 9743(2020)$.

356 23. Thomsen, M.B.H. et al. Comprehensive multiregional analysis of molecular 357 heterogeneity in bladder cancer. Sci Rep 7, 11702 (2017).

358 24. Chen, Z. et al. Single-cell RNA sequencing highlights the role of inflammatory cancerassociated fibroblasts in bladder urothelial carcinoma. Nat Commun 11, 5077 (2020).

360 25. Korsunsky, I. et al. Fast, sensitive and accurate integration of single-cell data with Harmony. Nat Methods 16, 1289-1296 (2019).

26. Stuart, T. et al. Comprehensive Integration of Single-Cell Data. Cell 177, 1888-1902 e21 (2019).

364 27. McGinnis, C.S., Murrow, L.M. \& Gartner, Z.J. DoubletFinder: Doublet Detection in Single-Cell RNA Sequencing Data Using Artificial Nearest Neighbors. Cell Syst 8, 329-

28. Yu, Z. et al. Single-Cell Transcriptomic Map of the Human and Mouse Bladders. J Am Soc Nephrol 30, 2159-2176 (2019).

29. Kim, S. et al. Epigenetic regulation of mammalian Hedgehog signaling to the stroma determines the molecular subtype of bladder cancer. Elife 8(2019).

371 30. Granja, J.M. et al. ArchR is a scalable software package for integrative single-cell chromatin accessibility analysis. Nat Genet 53, 403-411 (2021). 
373 31. Zhang, S., Yang, L., Yang, J., Lin, Z. \& Ng, M.K. Dimensionality reduction for single cell RNA sequencing data using constrained robust non-negative matrix factorization. NAR Genom Bioinform 2, lqaa064 (2020).

376 32. Lee, D.D. \& Seung, H.S. Learning the parts of objects by non-negative matrix factorization. Nature 401, 788-91 (1999).

378 33. Yin, Y. et al. Impact of cytosine methylation on DNA binding specificities of human transcription factors. Science 356(2017).

34. Herrmann, C., Van de Sande, B., Potier, D. \& Aerts, S. i-cisTarget: an integrative genomics method for the prediction of regulatory features and cis-regulatory modules. Nucleic Acids Res 40, e114 (2012).

35. Kim, E. et al. Creation of bladder assembloids mimicking tissue regeneration and cancer. Nature 588, 664-669 (2020).

36. Oh, D.Y. et al. Intratumoral CD4(+) T Cells Mediate Anti-tumor Cytotoxicity in Human Bladder Cancer. Cell 181, 1612-1625 e13 (2020).

37. Simoni, Y. et al. Bystander CD8(+) T cells are abundant and phenotypically distinct in human tumour infiltrates. Nature 557, 575-579 (2018).

38. Di Pilato, M. et al. CXCR6 positions cytotoxic T cells to receive critical survival signals in the tumor microenvironment. Cell 184, 4512-4530 e22 (2021).

39. Tang, Q. et al. TM4SF1 promotes EMT and cancer stemness via the Wnt/betacell lung cancer proliferation, invasion and chemo-resistance through regulating the DDR1/Akt/ERK-mTOR axis. Respir Res 20, 106 (2019). 
41. Gao, H. et al. Multi-organ Site Metastatic Reactivation Mediated by Non-canonical Discoidin Domain Receptor 1 Signaling. Cell 166, 47-62 (2016).

42. Bae, S. et al. Combined omics analysis identifies transmembrane 4 L6 family member 1 as a surface protein marker specific to human mesenchymal stem cells. Stem Cells Dev 20, 197-203 (2011).

43. Zacharias, W.J. et al. Regeneration of the lung alveolus by an evolutionarily conserved epithelial progenitor. Nature 555, 251-255 (2018).

44. Prasetyanti, P.R. \& Medema, J.P. Intra-tumor heterogeneity from a cancer stem cell perspective. Mol Cancer 16, 41 (2017).

45. Zhang, Y. et al. Cancer stem-like cells contribute to cisplatin resistance and progression in bladder cancer. Cancer Lett 322, 70-7 (2012).

46. Xiong, Y. et al. KNSTRN promotes tumorigenesis and gemcitabine resistance by activating AKT in bladder cancer. Oncogene (2021).

47. McLaren, W. et al. The Ensembl Variant Effect Predictor. Genome Biol 17, 122 (2016).

48. Cingolani, P. et al. Using Drosophila melanogaster as a Model for Genotoxic Chemical Mutational Studies with a New Program, SnpSift. Front Genet 3, 35 (2012).

49. Dunn, T. et al. Pisces: an accurate and versatile variant caller for somatic and germline next-generation sequencing data. Bioinformatics 35, 1579-1581 (2019).

50. Talevich, E., Shain, A.H., Botton, T. \& Bastian, B.C. CNVkit: Genome-Wide Copy Number Detection and Visualization from Targeted DNA Sequencing. PLoS Comput Biol 12, e1004873 (2016).

51. Venkatraman, E.S. \& Olshen, A.B. A faster circular binary segmentation algorithm for the analysis of array CGH data. Bioinformatics 23, 657-63 (2007). 
419 52. Chen, S., Zhou, Y., Chen, Y. \& Gu, J. fastp: an ultra-fast all-in-one FASTQ preprocessor.

420

421

422

423

424

425

426

427

428

429

430

431

432

433

434

435

436

437

438

439

440 Bioinformatics 34, i884-i890 (2018).

53. Zhang, Y. et al. Model-based analysis of ChIP-Seq (MACS). Genome Biol 9, R137 (2008).

54. Love, M.I., Huber, W. \& Anders, S. Moderated estimation of fold change and dispersion for RNA-seq data with DESeq2. Genome Biol 15, 550 (2014).

55. Reilly, S.K. et al. Evolutionary genomics. Evolutionary changes in promoter and enhancer activity during human corticogenesis. Science 347, 1155-9 (2015).

56. Ramirez, F., Dundar, F., Diehl, S., Gruning, B.A. \& Manke, T. deepTools: a flexible platform for exploring deep-sequencing data. Nucleic Acids Res 42, W187-91 (2014).

57. Yu, G., Wang, L.G. \& He, Q.Y. ChIPseeker: an R/Bioconductor package for ChIP peak annotation, comparison and visualization. Bioinformatics 31, 2382-3 (2015).

58. Bray, N.L., Pimentel, H., Melsted, P. \& Pachter, L. Near-optimal probabilistic RNA-seq quantification. Nat Biotechnol 34, 525-7 (2016).

59. Azizi, E. et al. Single-Cell Map of Diverse Immune Phenotypes in the Breast Tumor Microenvironment. Cell 174, 1293-1308 e36 (2018).

60. Jin, S. et al. Inference and analysis of cell-cell communication using CellChat. Nat Commun 12, 1088 (2021).

61. Welch, J.D. et al. Single-Cell Multi-omic Integration Compares and Contrasts Features of Brain Cell Identity. Cell 177, 1873-1887 e17 (2019).

62. Angerer, P. et al. destiny: diffusion maps for large-scale single-cell data in R. Bioinformatics 32, 1241-3 (2016). 
441 63. Bergen, V., Lange, M., Peidli, S., Wolf, F.A. \& Theis, F.J. Generalizing RNA velocity to transient cell states through dynamical modeling. Nat Biotechnol 38, 1408-1414 (2020).

443 64. Yan, H., Gunawan, A.D.M. \& Zhang, L. S-Cluster++: a fast program for solving the cluster containment problem for phylogenetic networks. Bioinformatics 34, i680-i686

445 (2018).

446 65. Giansanti, V., Tang, M. \& Cittaro, D. Fast analysis of scATAC-seq data using a predefined set of genomic regions. F1000Res 9, 199 (2020).

448 66. Gulati, G.S. et al. Single-cell transcriptional diversity is a hallmark of developmental 449 potential. Science 367, 405-411 (2020).

450 67. Smyth, G.K. Linear models and empirical bayes methods for assessing differential 451 expression in microarray experiments. Stat Appl Genet Mol Biol 3, Article3 (2004).

452 68. Hanzelmann, S., Castelo, R. \& Guinney, J. GSVA: gene set variation analysis for microarray and RNA-seq data. BMC Bioinformatics 14, 7 (2013).

454 69. Benaglia, T., Chauveau, D., Hunter, D.R. \& Young, D.S. mixtools: An R Package for Analyzing Finite Mixture Models. Journal of Statistical Software 32, 1-29 (2009). 


\section{Methods}

$458 \quad$ Human biospecimens and cell lines

459 This study was conducted in accordance with the measures of China on the administration of

460 clinical research, the Declaration of Helsinki, and the Institutional Ethic Protocols of Zhongnan

461 Hospital of Wuhan University (ZHWU), Beijing Friendship Hospital, Peking University

462 International Hospital and Hubei Cancer Hospital. The study protocol was approved by the

463 Institutional Review Board (IRB) at ZHWU (approval number: 2015029). All patients and all

464 relatives of organ donors provided written informed consent. All study procedures were performed

465 in accordance with the ethical standards of the Institutional Ethics Review Committee. Human

466 sample preservation by the Department of Biological Repositories at ZHWU, the official member

467 of the International Society for Biological and Environmental Repositories

468 (https://irlocator.isber.org/details/60), was approved by the IRB (approval number: 2017038) at

ZHWU and China Human Genetic Resources Management Office, Ministry of Science and

470 Technology of China (approval number: 20171793).

471

472 Clinical, pathological, and follow-up data records for all patients were collected (Supplementary

473 Table 1) and three pathologists were invited to independently confirm the histology diagnosis.

474 Additional cancer patient samples were collected through the clinical practice of the ZHWU,

475 Beijing Friendship Hospital, Peking University International Hospital and Hubei Cancer Hospital.

476 The study uses of clinical information and human samples (including blood, surgical tissue

477 specimens, and primary cancer cells) was approved by the Institutional Ethics Review Committee

478 (approval number: 2015029). Routine laboratory tests and pathology assessments were done

479 according to the relevant Chinese clinical protocols. 
RT4 and 5637 cells were kindly provided by Cell Bank, Chinese Academy of Sciences (Shanghai,

AR and RT4-AR stable cell lines, the LV6-AR lentivirus packaging was provided by GenePharma supplement polybrene. After two rounds of infection, cells were selected with $1 \mu \mathrm{g} / \mathrm{ml}$ puromycin. (https://uroweb.org/individual-guidelines/oncology-guidelines/). Human peripheral blood was collected with BD EDTA tube according to the manufacturer's protocol and stored at 4 degree (PBMC) was separated with the standard Ficoll protocol. Fresh tumour or normal tissues was collected during surgery and transferred to laboratory in high glucose, 10\% FBS supplemented DMEM. Tissue sample was resected in PBS prior to single cell dissociation. For methylation sequencing, tissue samples were flash frozen in liquid nitrogen and stored at -80 degree Celsius.

\section{Public data}

496 The study used a series of human bladder cancer sequencing data from NCBI SRA sequencing 497 read archive (https://www.ncbi.nlm.nih.gov/sra) with the accession code PRJNA662018. TCGA BLCA dataset from UCSC XENA (http://xena.ucsc.edu) were used in the study. The IMVigor210 dataset were downloaded from http://research500 pub.gene.com/IMvigor210CoreBiologies/packageVersions/. The Encode transcription factor

501 binding sites, ChromHMM tracks, $\mathrm{CpG}$ islands, repeat regions data, and lift-over chains were 502 collected from UCSC Genome Browser (http:/www.genome.ucsc.edu/). Transcription factor 
binding information were downloaded from ReMap database (http://pedagogix-tagc.univmrs.fr/remap).

\section{Statistical methods}

No statistical methods were used to determine the sample size used in the study. Between-group statistics was done with T-test (two-sided) or Wilcoxon Rank Test if T-test was not applicable. Survival analysis was done by Cox method. Detailed statistical methods were briefly denoted in the figure legends or text accompanying. All statistical analysis in this study were performed using R (3.6.2) (http://CRAN.R-project.org).

\section{Molecular Biology}

\section{Nucleic acid preparation}

For methylation sequencing or mutation panel capture sequencing, genomic DNA was extracted from tumour or normal tissue with Qiagen Animal Tissue DNA Extraction Kit (Qiagen Cat. \#69504) according to the manufacturer's protocol. Genomic DNA from FFPE tissue slides were extracted using MagPure Tissue DNA DF Kit (Magen Inc., Cat. \#MD5112-TL-06). Extracted DNA were quality-controlled by Qubit dsDNA HS assay (Thermo Fisher Scientific) and Agilent 2100 Fragment Analyzer.

\section{Chromatin accessibility sequencing (ATAC-seq) on tumour tissue}

20mg flash-frozen tumour tissue samples were minced using a double-sized douncer (Sigma, Cat. \#D8938) in 1xHB (0.25M sucrose, 0.06M KCl, 0.015M NaCl, 0.005M MgCl2, 0.01M Tris-HCl $\mathrm{pH} 7.5$ ), added to $5 \mathrm{ml}$ trypsin and $40 \mu 1$ 5U/ $\mu 1$ DNase I (Sigma, Cat. \#D5025) and digested in 37 
degree Celsius for 45 mins, with two times of rotation in between to mix the reaction. The digested cells were then neutralized with equal volume DMEM (Thermo Fisher, Cat. \#11995065) plus 10\% FBS (Gibco, Cat. \#16000044) and filtered through a 70 $\mu \mathrm{m}$ cell filter (BD Falcon, Cat. \#352350). The homogenize was centrifuged at 500x g, 4 degree Celsius for 5 mins. The sedimented cells were then resuspended in $400 \mu \mathrm{l} 1 \mathrm{xHB}$ and washed once, transferred to $2 \mathrm{ml}$ LoBind Tube (Eppendorf) and washed again. Cells were counted using Trypan blue (Solarbio, Beijing, China). After quantification, the cells were then added to a 30\%-40\%-50\% iodixanol (Sigma, Cat. \#D1556) gradient and centrifuged at $3000 \mathrm{~g}, 20$ mins at 4 degree Celsius. The cell layer at 30\%-40\% interface was collected for library preparation. DNA library were prepared ('tagmentation') with a Tn5 transposase kit (Vazyme, Cat. \#TD501) using 1 million cells per reaction according to manufacturer's protocol. After tagmentation and PCR amplification, the sequencing library were quality-controlled with SYBR-green based qPCR using primers for house-keeping gene (GAPDH) promoter and gene desert (chr5: 105187030-105190000) before sequencing.

\section{Cut\&Tag sequencing on cells}

RT4 or 5637 cells were counted using Trypan blue (Solarbio, Beijing, China). After quantification, 40 million cells were used for Cut\&Tag experiment. Cut\&Tag experiment were performed with NovoProtein Cut\&Tag 2.0 pAG-Tn5 kit (NovoProtein, Cat. \#N259) according to the manufacturer's protocol. Antibodies used in this study include: anti-H3K4me3 (Diagenode, Cat. \#C154100003), anti-H3K27me3 (Abcam Cat. \#ab6002), anti-H3K27ac (Abcam Cat \#ab4729), anti-FOXA1 (Abcam Cat. \#ab170933) and anti-AR (Abcam Cat. \#ab108341), and Goat-antimouse IgG (Sangon, Cat. \#D111024) and Goat-anti-rabbit IgG (Sangon, Cat. \#D111018). Each library was sequenced to $2 \mathrm{x}$ human genome coverage on Novaseq sequencer (Illumina, CA). 


\section{Single stranded DNA methylation capture sequencing}

Tissue genomic DNA (200ng) were bisulfite converted using EZ-DNA Methylation-Gold Kit

552 (Zymo Research, Cat. \#D5006) according to the manufacturer's protocol. After conversion, the DNA were subjected to a single-stranded library preparation protocol Tequila 7N (Euler overhang adaptor using T4 DNA ligase (Enzymatics), and linearly amplified for 12 cycles using adaptor with 7bp 3' random nucleotide overhang and PCR-amplified using adaptor oligos (Sangon, $100 \mathrm{M}$ paired-end $150 \mathrm{bp}$ reads.

\section{DNA mutation panel sequencing}

567 DNA was sonicated into 250 bp fragments with Covaris S220. NGS sequencing libraries were 568 built with a single-stranded DNA ligation protocol. In brief, sonicated DNA was denatured to form 569 a single strand and 3'-polyA-tailing was performed with terminal transferase (Cat. \#P7070, 570 Enzymatics Ltd., USA). Ligation of a polyT-extruding adaptor (Sangon Ltd., China) was 571 performed with E.coli ligase (Cat. \#2161, Takara Ltd., Japan). Linear amplification of the ligated 
572 product was performed with adaptor-specific primer (Sangon Ltd., China) for 12 cycles and the

573 amplified product was annealed and ligated into a 5'-polyN-extruding adaptor (Sangon Ltd., China)

574 with T4 ligase (Cat. \#L6030, Enzymatics, USA). The ligated product was then amplified with

575 Illumina-compatible primers (Sangon Ltd., China) for 10 cycles. For panel capture sequencing,

576 the amplified library was captured using either: (1) a custom-synthesized oncology panel-

577 consisting exons, UTR and structural variant breakpoint-enriched introns of 538 tumour-related 578 genes, as well as 1076 SNP loci (Euler Technology Ltd., China), or (2) a custom-synthesized whole

579 exon panel consisting exons, UTR and SNP loci from GRCh37 version human genome totalling 580 52.6Mbp (Euler Technology Ltd., China). Libraries were sequenced to targeting $\sim 800 \mathrm{x}$ (oncology panel) or $\sim 150 \mathrm{x}$ (whole-exome) on-target coverage with paired-end 150bp read format on Illumina Novaseq.

\section{Single cell RNA and chromatin accessibility sequencing}

Fresh tissues were processed immediately after being obtained from bladder cancer patients.

Tissues were cut into tiny pieces $(<1 \mathrm{~mm}$ diameter) and then subjected to dissociation using collagenase II (Biofrox, Cat. \#2275MG100) and $100 \mu$ l of DNase (Servicebio, Cat. \#1121MG010) subsequently centrifuged at $250 \mathrm{~g}$ x $5 \mathrm{~min}$. Cell pellets were washed in PBS twice, and resuspended in $1 \mathrm{ml}$ ice cold RBC lysis buffer and incubated at 4 degree Celsius for 10 mins. $10 \mathrm{ml}$ of ice-cold PBS was added to the tube and subsequently centrifuged at $250 \mathrm{~g}$ x $10 \mathrm{~min}$. After decanting the supernatant, the pellet was resuspended in $5 \mathrm{ml}$ of calcium- and magnesium- free PBS containing 0.04\% weight/volume BSA. Cells were counted using Trypan blue (Solarbio, Beijing, China). For 594 chromatin accessibility sequencing, approximately $10^{6}$ cells were used for nucleus extraction. 
595 Nucleus extraction were performed as 10x single cell library preparation was done according to 596 the manufacturer's protocol. Chrominum Single Cell 3' V3 kits, 5' V3 kits (for TCR sequencing), 597 and ATAC V2 kits were used. For RNA sequencing, single-cell suspensions were loaded onto a 598 Chromium Single-Cell Controller Instrument (10× Genomics) to generate single-cell gel beads in 599 emulsions (GEMs) targeting $~ 8000$ cells (3'/5' RNA). After generation of GEMs, reverse 600 transcription reactions were engaged to generate barcoded full-length cDNA, which was followed 601 by disruption of emulsions using the recovery agent, and then cDNA clean-up was performed with 602 DynaBeads Myone Silane Beads (Thermo Fisher Scientific). Next, cDNA was amplified by PCR. 603 Subsequently, the amplified cDNA was fragmented, end-repaired, A-tailed, and ligated to an index 604 adaptor, and then the library was amplified. To amplify TCR sequence, 10x Genomic TCR kit was 605 used according to the manufacturer's protocol. The scRNA libraries were sequenced aiming to 606 have $\sim 5000$ reads per cell on Illumina Novaseq with paired-end 150bp reads.

607

608 For ATAC, tagmentation were performed according to the manufacturer's protocol. After 609 tagmentation reaction, nucleus suspensions were loaded a Chromium Single-Cell Controller 610 Instrument $(10 \times$ Genomics $)$ targeting $~ 10000$ nucleus in one reaction. After generation of GEMs, 611 PCR reaction were performed to amplify the library. DNA clean-up was performed with size612 selection XP beads. Libraries were sequenced aiming to have $~ 5000$ reads per cell on Illumina 613 Novaseq with paired-end 50bp reads.

614

615 Bioinformatics

$616 \quad$ Mutation profiling 
617 Raw sequencing data were mapped using bwa-mem to GRCh37 reference genome with default

618 parameters. Germline mutations were called with the Sentieon haplotyper (Sentieon-Genomics-

619 201808.05) and annotated with $\operatorname{VEP}^{47}$ (90.1) and $\operatorname{SnpSift}^{48}$ (4.2). For paired tumour-normal

620 samples, candidate germline variants were filtered with the gnomAD global frequency $<0.001$ and

621 in-house database frequency $<0.001$ (out of 20,000 patients). Intersection with variants found in

622 different male members of the pedigree was performed to extract patient-specific germline

623 mutations. Somatic tumour mutations were called with the Sentieon TNscope (Sentieon-

624 Genomics-201808.05) with paired sample or NA12878 (at unpaired cases) and Pisces ${ }^{49}(5.2 .9 .122)$,

625 while variants called by both algorithms were passed for filtering. CNV were called using

$626 \mathrm{CNVkit}^{50,51}$ with default parameter. B-allele frequency (BAF) determination was performed for

627 germline and somatic variants. Tumour genome was segmented using BAF and sequencing depth

628 information. Allelic copy numbers were determined for each somatic variant using a

629 hypergeometrical test. Tumour content determined by an in-house CNV-based linear regression

630 method was confirmed by Hematoxylin and Eosin (H\&E) staining. Minimal tumour-cell-fraction

631 of $5 \% / 2 \%$, minimal variant read number of $10 / 3$, and minimal read depth of 500/30 were applied

632 to variants for filtering for panel sequencing or whole-exon sequencing, respectively. Filtered

633 mutations were annotated with vefanno and filtered with gnomAD global frequency $<0.001$.

634

635 Genomic region liftover

636 For genomic regions liftover between UCSC GRCh38 and GRCh37, the R package easyLift 637 (https://github.com/caleblareau/easyLift), the liftover executable from UCSC Kent Utility 638 (https://genome.ucsc.edu/cgi-bin/hgLiftOver) and the lift-over synteny chain files from UCSC

639 Genome Browser were used. Target genome was always GRCh38. 


\section{DNA methylation data processing}

642 Raw bisulfite-converted DNA methylation sequencing data, either downloaded from NCBI SRA

643 or directly from in-house sequencing, were processed using fastp (--trim-front2 20 -w 20) ) $^{52}$

644 (https://github.com/OpenGene/fastp) and mapped to GRCh37+decoy reference genome using 645 BWA-Meth (https://github.com/brentp/bwa-meth) using standard parameters. Mapped data were 646 deduplicated and sorted using Sambamba (https://github.com/biod/sambamba) and Samblaster 647 (https://github.com/GregoryFaust/samblaster). CpG-methylation level was extracted using Pile-O648 Meth (https://github.com/dpryan79/MethylDackel) toolkit. For all libraries, conversion rate was quality controlled by $\mathrm{CHH}$ methylation level $>99 \%$. Basic statistics of in-house sequencing library were further quality-controlled by on-target rate and on-target coverage with bedtools (https://github.com/arq5x/bedtools), and duplication rate and mapping rate with Sambamba.

\section{Differential methylated loci and region}

$654 \mathrm{CpG}$ methylation level (beta: defined as reads of $\mathrm{C}$ nucleotide over total read coverage on single difference between case and control groups $>0.1$. Initial DMR candidate were made by merging within-100bp-apart DML. The average beta of each initial DMR were calculated as mean beta of all $\mathrm{CpG}$ encompassed in the DMR. This average beta was subjected to t-test and $\mathrm{P}<0.01$ regions 
using a circular binary segmentation approach on beta difference case and control groups with DNAcopy $^{51}$ (https://github.com/veseshan/DNAcopy). K-means clustering was performed using $\mathrm{R}$ (3.6.2) on the methylation beta difference on each segment, and clusters of segments fully encompassed candidate 'seed' DMR were selected as true DMR candidate.

\section{ATAC and Cut\& Tag sequencing data preprocessing}

Raw paired-end open chromatin tagmentation (ATAC) and Cut\&Tag sequencing data were mapped to human reference genome GRCh38 using Bowtie2 (-k 10 --very-sensitive -X 2000) (https://github.com/BenLangmead/bowtie2). All unmapped reads, non-uniquely mapped reads, reads with low mapping quality $(\mathrm{MAPQ}<20)$ and PCR duplicates were removed. For Cut\&Tag sequencing libraries, data were used as is. For in-house prepared ATAC-seq data, the data were quality-controlled by assessing insertion size (using an in-house R script) and TSS-enrichment (using an in-house $\mathrm{R}$ script with GenomicRanges package (https://github.com/Bioconductor/GenomicRanges) measuring the depth ratio at the promoter region (GRCh38 refFlat annotation from UCSC Genome Browser) (0bp of TSS vs. 1kbp +- of TSS). A QC-passed ATAC-seq library must have TSS enrichment of 6, mapped deduplicated sequencing fragments $\geq 20 \mathrm{M} \quad \mathrm{PE} \quad$ reads, $\quad$ PCB1 $>0.9, \quad$ PCB2 $>3$ (https://www.encodeproject.org/pipelines). Enrichment peaks were determined by intersecting peaks found from MACS2 callpeak (-f BAMPE) ${ }^{53}$ (https://github.com/taoliu/MACS) and Genrich $\left(\begin{array}{lllllllll}-r & -m & 1 & -j & \text { for } & \text { ATAC } & \text { only; and standard parameter for Cut\&Tag) }\end{array}\right.$ (https://github.com/jsh58/Genrich). Quality control of ATAC-seq libraries including read length, V-plot and TSS-enrichment were done with custom $\mathrm{R}$ script and deeptools (https://github.com/deeptools/deepTools). Reliable peaks were identified with IDR 
686 (https://www.encodeproject.org/software/idr). Reliable ATAC peaks from different set of data

687

688

689

690

691

692

693

694

695

696

697

698

699

700

701

702

703

704

705

706

707

were converged with $1 \mathrm{bp}$ minimum overlap and extended to the largest width of overlapping peaks.

Joining these operation results in a set of non-overlapping, varied-width peaks across the genome encompassing all reliable open chromatin region.

\section{Measurement of difference of ATAC-seq peaks}

Read coverage of sequencing library were collected over the repeatable ATAC enrichment peak mentioned above with Sambamba. Differential enrichment was performed with DESeq $2^{54}$ (https://github.com/mikelove/DESeq2) using standard parameters. For samples with few replicates, we adapted a general linear model approach for estimating difference following the method mentioned in Reilly et al $2015^{55}$.

\section{Classification of differentially methylated region by Cut\&Tag signal}

Reads from Cut\&Tag sequencing library were transformed into bigwig using deepTools ${ }^{56}$. Windows $+-1200 \mathrm{bp}$ around the differentially methylated loci were used to extract the reads. The resulting coverage matrix containing $\mathrm{H} 3 \mathrm{~K} 27 \mathrm{me} 3$, H3K27ac, H3K4me3, anti-FOXA1 and anti-AR signals were then clustered using K-means with deepTools ${ }^{56}$. Annotation of these K-means clustered regions were done by ChIPseeker ${ }^{57}$ using $-1000 \mathrm{bp} \sim+500 \mathrm{bp}$ as promoter around TSS, and their relative distribution on different types of genomic regions were plotted using pheatmap (https://cran.r-project.org/web/packages/pheatmap/index.html). Regions were labelled 'Promoter' if they were mainly covered by H3K4me3 in any cell type, 'Enhancer' if they were covered by H3K27ac in any cell type, and 'Repressor' if they were covered by H3K27me3 in any cell type. 
708

709

710

711

712

713

714

715

716

717

718

719

720

721

722

723

724

725

726

727

728

729

730

Upon these regions we did not detect bivalent promoter/enhancer features with H3K4me3/H3K27me3 double positivity.

\section{Single cell RNA preprocessing}

Loompy-Kallisto ${ }^{58}$ were used for mapping the RNA data for gene expression analysis. Loom files were read in $\mathrm{R}$ by hdf5r (https://cran.r-project.org/web/packages/hdf5r/index.html) and preprocessed with Seurat ${ }^{26} 3.0$. Quality control was done for every single sample individually to filter against gene counts, UMI counts, total reads, mitochondrial reads. Generally, cells with $>10 \%$ mitochondrial reads, or with UMI $<600$ or $>5000$, or with gene counts $>5000$ were filtered prior to subsequent analysis. Such quality control process might iterate at every subsequent step to ensure the stringency of analysis. Individual samples were processed through the Seurat pipeline.

Data firstly passed DoubletFinder ${ }^{27}$ pipeline with standard parameters to filter against potential doublets. The filtered data were then normalized and top 2000 variable genes were identified. Ribosomal proteins, heat shock proteins, and chaperones were intentionally removed from the variable gene list because of the highly inconsistent nature of their behaviours between different tissue types. Gene expression profile were then scaled and reduced by PCA using Seurat. Generally, $\geq 30$ PCA components were included in subsequent steps. FindClusters function were initially performed using a high resolution then gradually lowered to ensure the final clusters are less than PC components. SingleR annotation with human reference and conventional markers were used to categorize the cell clusters. Differentially expressed genes were identified with Seurat FindAllMarkers function with Wilcoxon test for the cell clusters. Comparison of the found DE genes with conventional markers were performed to ensure that clusters contain relatively pure cell population. Cell-type-annotated cells were then separated into different subsets based on their 
731 types. In this study, epithelial cells, endothelial cells, fibroblast, CD8 T cells, CD4 T cells, NK

732

733

734

735

736

737

738

739

740

741

742

743

744

745

746

747

748

749

750

751

752

753

cells, B cells and myeloid cells were considered 'pools' for subsequent analysis. After pre-

processing, similar type of cells from different samples were merged and re-analyzed. Quality control, doublet identification, dimensionality reduction, cluster identification, differentially expressed gene identification, and cell type identification were iteratively performed on these 'pure' sets of cells. In such setting, cell type composition between samples were relatively homogeneous and usually it is unnecessary to perform data integration. When sample-driven variation is evident or in cases when dataset contains both $3^{\prime}$ and 5' scRNA samples, to control against technical variations, Harmony ${ }^{25}$ were applied to datasets with (vars.to.regress = 'chemistry') option during data scaling. 'Contaminant' cells which mis-segregated into large pools were identified and put back into the unprocessed pool. Iterative processing of cells was done semi-automatically until all cells were processed. In the end, a total of 133953 cells (61724 epithelial, 5458 endothelial, 15203

fibroblast, 1918 myeloid, $23070 \mathrm{CD} 4,12165 \mathrm{CD} 8,783 \mathrm{NK}$ and $13632 \mathrm{~B}$ cells) were collected for downstream study. Data were visualized with 2D tSNE or UMAP projection.

\section{TCR integration}

TCR sequencing data were processed with cellranger-tcr ${ }^{59}$ with standard parameters. After barcode matching and TCR de novo assembly, the assembled TCR sequence and clonotypes were extracted. Clones of cells with similar TCR in the same donor were identified as having exactly similar TCR exon usage combination as well as similar CD3r nucleotide sequence for all TCR (alpha and beta chain, if applicable) sequences expressed in the cell. Cells with $\geq 3$ TCR sequences were removed. Clonal expansion is defined as a single $\mathrm{T}$ cell clone was observed $>2$ times in sequencing data from the donor. Local clonal expansion was defined as the clone is observed $>2$ times in one sample 
754

755

756

757

758

759

760

761

762

763

764

765

766

767

768

769

770

771

772

773

774

775

776

and $<2$ times in all other samples. Global clonal expansion was defined as the clone is observed $>2$ times in $\geq 2$ samples. scRNA data were subsequently registered with this TCR information to annotate the clonal identity and clonal expansion status of each T lymphocyte.

\section{Cell-cell signalling inference}

Cell-cell signalling inference was done with CellChat ${ }^{60}$ with cells from normal bladder, bladder cancer tissue, adjacent bladder tissue and metastasis prostate tissue. Cell types with $\leq 20$ cells were excluded from the analysis. Signalling probability was extracted from the object.

\section{Co-regulated transcription module inference using NMF}

Non-negative matrix factorization was performed in R using the iterative NMF (iNMF) internal function from Liger ${ }^{61}$ with data scaled by sample. After NMF, the weight matrix, variation matrix, and loading matrix were extracted from the object. Prediction of metagene expression in single cell was done with the top 100 contributors of NMF component using Seurat AddModuleScore function.

\section{Transcription factor inference with cisTarget}

RcisTarget ${ }^{34}$ was used to extract enriched TFBS in the co-regulated gene modules from NMF (top 100 genes from each component) with the human GRCh38 -1000bp +500bp database downloaded from cisTopic (hg38_refseq-r80_500bp_up_and_100bp_down_tss.mc9nr.feather). After extraction, high confidence co-regulator TF of regulons with NES $>3.0$ were extracted from the data. Specific TF regulating a NMF component was defined as a TF which was found in $\leq 2$ components. TF binding affected by methylation was identified as the binding site is derived from 
777 the in vitro DNA-binding activity from Yin et al's work ${ }^{33}$. Visualization of co-regulated

778 transcription network is performed with visNetwork (https://cran.r-

779 project.org/package=visNetwork). Matching of TF to cell-cell signalling event was done manually.

\section{Developmental lineage analysis}

Diffusion map of single cell RNA expression data was computed with destiny ${ }^{62}$. RNA velocity analysis of single cell 3' RNA sequencing data was performed with scVelo ${ }^{63}$ using Reticulate $^{64}$ in R (3.6.2) with Python 3. With RNA velocity defined the root cluster, slingshot was performed on diffusion maps to produce minimally spanning tree lineages.

\section{Single cell ChrAcc data analysis}

Single cell ATAC (chromatin accessibility, scATAC) raw reads were mapped with cellranger-atac ${ }^{65}$.

The mapped fragment files were then processed by $\mathrm{ArchR}^{30}$. Quality control, doublet identification,

ArchR using Seurat CCA algorithm, with constrains for integration on large pools of cell type. 
800

801

802

803

804

805

806

807

808

809

810

811

812

813

814

815

816

\section{Cell cycle scoring}

818 Single cell RNA cell cycle scoring was done with Seurat ${ }^{26}$ using the 'cc.gene.2019' dataset as were added together to suggest the relative 'actively cycling' probability.

number varied regions were used as a mask for a second round of LSI-based dimensionality reduction to perform copy-number-invariant clustering of cells. Peaks were called for single cell dataset using MACS2. Chromatin accessibility on given genomic regions was also calculated in ArchR for single cell chromatin accessibility difference analysis. Transcription factor footprinting (activity measurement) was done in ArchR with ChromVar. Co-accessible regions were calculated with ArchR with `addCoAccessibility` (maxDist $=1000000)$ and filtered with correlation $>0.1$. To visualize scATAC coverage on given DMR regions, grouped single cell coverage files were written by ArchR and profile plots were made with deepTools 'plotProfile'.

\section{Epigenotype}

MIBC-specific DMR and MIBC differential ATAC peaks were collected and merged. The merged 'epigenetically change regions' were then used as regions for dimentionality reduction with LSI in ArchR. Epigenotype clusters were then subjected to a further dimensionality reduction with diffusion map, and slingshot lineage inference. Epigenotype match to RNA cluster (phenotype) was counted in R. The Shannon entropy of epigenotype-phenotype match was calculated to infer the transcriptional plasticity of given epigenotype. source of G2/M and S-phase gene sets. For visualization, G2M.score and S.score of a single cell

821

\section{Differentiation potential assessment}


823 Cellular differentiation potential were assessed by CytoTRACE ${ }^{66}$. Briefly, RNA expression matrix

824 of single cells are extracted from Seurat object, with all transcripts regardless of their in-assay-

825 variability. CytoTRACE analysis was performed on this matrix without downsampling. Per-cluster

826 median CytoTRACE score was used as an index for the differentiation state of each cell cluster.

827 For the comparison, we selected from the scRNA dataset all 'normal' urothelium cells, and cancer

828 cells which projected to scATAC counterparts. Cancer scRNA cells were labelled by their projected 829 scATAC counterparts.

830

831

SNV matching in single cell sequencing data

832

For mutation expression

analysis,

standard

cellranger

pipeline

(https://support.10xgenomics.com/single-cell-gene-expression/software/downloads/latest) were

used to produce a bam file from scRNA sequencing data. SNV were extracted from paired tumour

mutation panel capture sequencing results and lifted-over to hg38 using easyLiftOver based on

LiftOver. Only somatic mutations with $>5 \%$ VAF were included in the analysis. Vartrix (https://github.com/10XGenomics/vartrix) was used to build a cell $\mathrm{x}$ mutation matrix

838 (Supplementary Table 10).

Metagene activity in bulk RNA sequencing data

841 Metagene were defined as the top 100 genes that contribute to a NMF component. Raw read count

842 matrix of bulk BLCA tumour tissue RNA sequencing were downloaded from UCSC XENA and

843 IMVigor210 sources. Limma ${ }^{67}$ was used to normalize the RNAseq count matrix, and GSVA ${ }^{68}$ was $^{2}$

844 used to score metagene activity. Patients/samples were classified as 'high'/'low' with respect to

845 that metagene activity using a 2-mode Gaussian distribution fitting with mixtools ${ }^{69}$. 


\section{Survival analysis}

848 Sample-centered clinical data were downloaded from UCSC XENA and IMVigor210 sources.

849 Survival plots and analysis were done with $\mathrm{R}$ package survplot

850 (http://www.cbs.dtu.dk/ eklund/survplot/).

851 


\section{Data Availability Statement}

853

854

855

856

857

858

859

860

861

862

863

864

865

866

867

868

869

870

871

872

873

874

All data, including raw data and clinical information, have been submitted to the Genome Sequence Archive for Human (http://bigd.big.ac.cn/gsa-human/) at the BIG Data Center, Beijing Institute of Genomics, Chinese Academy of Sciences, under the accession number HRA001225. The raw sequencing data and clinical information unique to an individual and require controlled access. The deposited and publicly available data are compliant with the regulations of the China Human Genetic Resources Management Office, Ministry of Science and Technology of China.

\section{Funding}

This study was supported by grants from the Chinese Central Special Fund for Local Science and Technology Development of Hubei Province (2018ZYYD023), Research Fund of Zhongnan Hospital of Wuhan University (ZNJC201915, SWYBK, SWYBK01, SWYBK02), Science and Technology Department of Hubei Province Key Project (2018ACA159), and Improvement Project for Theranostic Ability on Difficulty Miscellaneous Disease (Tumor) from National Health Commission of China (ZLYNXM202006). The funders played no role in the study design, data collection and analysis, decision to publish, or preparation of the manuscript.

\section{Acknowledgments}

The excellent technical assistance of Danni Shan, Shanshan Zhang, Wen Chen, Wan Xiang, Hongwei Peng and Zongning Zhou at ZNWH is gratefully acknowledged. We also would like to acknowledge the TCGA consortium and authors publicly sharing data used in our research. The authors disclose no writing assistance. 
875

876

877

878

879

880

881

882

883

884

885

886

887

888

889

890

891

892

893

\section{Author Contributions}

Conceptualization, Y.X., Y.Zhang, X.W.;

Methodology, Y.X., W.Jin, K.Q., K.W., H.Z., Y.W., F.C., Y.Zhang;

Computation and Formal analysis, W.Jin, K.W., L.S., F.C., Y.Zhang;

Investigation, Y.X., W.Jin, K.Q., K.W., G.W., L.J., Y.Zhao, L.C., X.Chen, N.L., L.S., Sheng Li, H.S., M.P., Y.Zhang;

Resources, K.Q., G.W., R.C., L.J., Y.Zhao, H.Z., T.L., Z.X., T.W., J.L., F.Y., D.L., H.C., Z.Y., Sheng Li, H.S., Z.G., Y.G., N.L., S.J.L., W.D., W.Z., D.C., Y.T., Y.L., C.J., X.H., H.M., X.W.;

Writing Original Draft, Review, and Editing, Y.X., W.Jin, K.Q., G.W., W.Jiang, R.C., L.J., Y.Zhao, X.Cao, M.P., Y.Zhang, X.W.;

Writing Critical Review, Commentary or Revision, Y.X., K.Q., G.W., W.Jiang, L.J., Y.Zhang, X.W.;

Writing Data Presentation, Y.X., W.Jin, K.Q., G.W., W.Jiang, L.J., Y.Zhang;

Supervision, Z.X., Y.Zhang, X.W.;

Project Administration, Y.X., K.Q., Z.X., Shenjuan Li, Y.Zhang, X.W.;

Funding acquisition, Y.X., Y.Zhang, X.W.

\section{Competing interests}

The authors declare that they have no competing interests. 


\section{Figure legends}

896

897

898

899

900

901

902

903

904

905

906

907

908

909

910

911

912

913

914

915

916

917

\section{Figure 1. Single cell landscape of BLCA.}

(A) UMAP-projection of scRNA sequencing from bladder tissues. Epithelial cells, fibroblast (FB), endothelial cells (Endo), macrophage and monocyte (Myeloid), CD4 ${ }^{+}$helper T cells (CD4), CD8 ${ }^{+}$ cytotoxic T cells (CD8), natural killer cells (NK), and B lymphoid cells (B) were shown. (B) Marker gene expression for each cell type. (C) Cell type composition in bladder tissues of in-house sequenced sample donors. Donors were separated by T stage $(0$ : healthy organ donor; 0.5 : Ta; 1 4: T1-T4; Ta/T1: NMIBC; T2-T4: MIBC). (D) Tissue type prevalence for each donor. Donors were separated by clinical stage as in (C). (E) UMAP-projection of epithelial cells. Normal epithelial cells were identified by inferred CNV in (F). Fast-cycling, proliferating cancer cells were identified by cell cycle score (G). Luminal-like, basal-like, and TM4SF1-positive cancer cells were identified by marker gene expression (Supplementary Fig. 2). (F) Inferred CNV from integrated scATAC data. (G) Inferred cell cycle score. (H-M) UMAP-projection of CD8-positive cytotoxic T, CD4positive helper T, NK, B, Myeloid and Fibroblast (Supplementary Fig. 6-7 and 9-12). (N) Cell type prevalence in each donor. Bars are coloured as in panels $(\mathrm{H})-(\mathrm{M})$, respectively. Donors are separated by clinical stage as (C). Significant changes in cellular composition through clinical stage advancements were found for CD8 (recruitment of bystander $\mathrm{T}_{\text {eff }}$ ), CD4 (emergence of tumour-reactive $\operatorname{tr} \mathrm{T}_{\text {reg }}$ ), and fibroblasts.

\section{Figure 2. Bladder urothelium cell fate determination by mutual signalling.}

(A) TSNE-projection of normal bladder urothelium epithelial cells from the 3 healthy organ donors. BsP: basal progenitor cell $\left(\mathrm{SHH}^{+} / \mathrm{CD}_{4} 4^{+} / \mathrm{TM} 4 \mathrm{SF} 1^{+}\right) ; \quad \mathrm{Bs} 1:$ basal cell 1 $\left(\mathrm{IGFBP}^{+} / \mathrm{KRT}^{+} 7^{+} / \mathrm{PTGS}^{+}\right) ; \quad$ Bs $2:$ basal cell 2 (HES1 $\left.{ }^{+} / \mathrm{ELF}^{+}\right) ; \quad$ Bs3: basal cell 3 
$\left(\mathrm{HES1}^{+} / \mathrm{PCSK} 5^{+}\right) ; \mathrm{Im} 1$ : intermediate cell $1\left(\mathrm{TXNIP}^{+}\right) ; \operatorname{Im} 2$ : intermediate cell $2\left(\mathrm{MMP}^{+}\right) ; \operatorname{Im} 3$ : intermediate cell $3\left(\mathrm{GDF} 15^{+} / \mathrm{KRT}^{2} 3^{+}\right)$; Um: umbrella cell $\left(\mathrm{CD} 24^{+} / \mathrm{KRT} 18^{+}\right)$. (B) Developmental trajectory of urothelium on 3D diffusion map projection. Basal progenitor cell evolves into three lineages: Bs1, Bs2/Bs3, and Im1/2/3->Um. (C) Cell cycle score of each cluster in (A). (D) Marker gene expression of each cluster in (A). Note that HES1/VEGFA are only positive in BsP/Bs1/Bs2, whilst UPK2/3A/3B and PIGR are only positive in intermediate/umbrella cells. Umbrella cell is identified by CXCL6 expression. (E) NA expression of basal/intermediate markers (KRT5/CD44 as basal, and UPK1A/3A as intermediate markers) and Notch signalling pathway ligand/receptors. Note that whilst Notch downstream effector HES1/HES4 are upregulated in basal cells, the main source of Notch ligand DLL1/JAG1 comes from the underlying endothelial/fibroblast population.

(F) Cell-cell signalling probability between epithelial cells suggesting Bs1 provides HBEGF to all other cells, Bs2/Bs3 providing FGF, and intermediate cells contribute BMP/TGF $\beta$ ligands. (G) Anatomy of urothelium from the basal (bottom) side to the luminal (top) side shows cell types and the identified cell-cell signalling events between them. Basal/intermediate cell lineages are likely to be separated by Notch signalling activity strength, as basal cells are more proximal to the major Notch ligand provider.

Figure 3. Origin of muscle-invasive and TM4SF1-positive bladder cancer cells in urothelium. (A) Single cell chromatin accessibility on MIBC-specific differentially methylated regions (DMR) among stromal (2/3/1/4), normal epithelial (14), and cancer (5-13 and 15). MIBC-hypo DMR are closed in normal epithelial cells but open in cancer. MIBC-hyper DMR are only open in stromal and normal epithelia. (B-C) Chromatin accessibility of MIBC-hyper DMR (B) and MIBC-hypo DMR (C) in normal epithelial, cancer and TPCS. (D) Unsupervised hierarchical clustering of DMR 
941

942

943

944

945

946

947

948

949

950

951

952

953

954

955

956

957

958

959

960

961

962

963

methylation level (Z-scaled) of BLCA tissue samples on selected hypermethylated DMR. Samples were labelled by invasiveness (Invasive) and clinical stage (T). NMIBC is clearly separated from MIBC by hypermethylation in GPX4/PRKCZ/SHH/CD44 genes, and MIBC could be classified into 4 clusters by DMR methylation level. (E) RNA expression of DMR-associated genes in different types of urothelial cells. (F) UMAP-projection of normal urothelium basal cells (BsP, Bs1, Bs2, Bs3), early-stage (CNV-low) BLCA cells (Ca), TM4SF1-positive cancer cells (TPCS), and fast-proliferating cancer cells (FPC). (G) 3D diffusion map and principal curve analysis of cells in (F), showing that FPC and TPCS branched early in the development of BsP towards Bs2/3. (H) Cell cycle score of each cell population in (F). BsP, FPC and TPCS are major proliferating populations. (I) Marker gene expression for cell types in (F). TM4SF1 marks both BsP and TPCS. Whilst HES1/4 are high in all basal cell lineage, they are both lost in TPCS. In contrast, TPCS specifically upregulates multiple keratins (KRT5/6A/6B/14/16).

\section{Figure 4. Extrinsic and intrinsic determinants in TPCS transformation.}

(A) Cell-cell signalling probability between BLCA cells in (Fig. 2F). C: Cancer; F: FPC; T: TPCS. BMP signalling distinguished TPCS from cancer and FPC. (B-C) Non-negative matrix factorization (NMF) component ("metagene") expression in single cells. NMF components 6, 11 and 16 are selectively upregulated in TPCS. (D) Co-regulated transcription networks extracted from the NMF component by common transcription factor binding site in their promoters showing extracellular signalling event controlling them. Both NMF6 and 11 are controlled by BMP and Notch (co-regulated by SMAD4/SMAD5/ASCL2). NMF16 is controlled by SHH and intrinsically regulated by DNA methylation (co-regulated by NFIB/GLI1/MEF2C). For details of the putative 
964 965

966

967

968

969

970

971

972

973

974

975

976

977

978

979

980

981

982

983

984

985

986

regulatory TF-gene relationship, please refer to Supplementary Table 7. (E) TF binding activity (z) and expression (Exp) in normal, luminal cancer, basal cancer, and TPCS from scATAC data.

Figure 5. Epigenetic reprogramming in TPCS transformation.

(A-B) Cut\&Tag profiling on MIBC-hypo DMR (A) and MIBC-hyper DMR (B). Left: distribution of DMR clusters on regulatory genomic elements. DMR were K-means clustered by epigenetic modification. Right: Cut\&Tag profile of histone modification (H3K4me3, H3K27ac and H3K27me3) and AR/FOXA1 binding in each DMR clusters. Bar annotation on the left denotes regulatory elements (red, promoter; blue, enhancer and black, repressor) (C-D) scATAC coverage of hypoC3 (C) and hypoC7 (D). DMR in hypoC3 bound by FOXA1 in both basal and luminal cells but lost H3K27ac modification and AR binding in basal cells (red box in B). DMR in hypoC7 bound by FOXA1 and lost H3K27ac in basal cell. (E-F) Epigenetic landscape of HES4 in basal T1 cells and RT4 (E) as well as TPCS and 5637 cell (F). (G-H) Epigenetic landscape of TFAP2C in basal T1 cells and RT4 (G) as well as TPCS and 5637 cell (H). Epigenetic features in F-I includes: chromatin accessibility (blue), FOXA1 binding (grey), histone modification (H3K4me3, red; H3K27ac, orange; H3K27me3, black) and DNA methylation (beta), DNAm.

\section{Figure 6. Epigenotype of TPCS is phenotypically plastic.}

(A) Prevalence of 'epigenotype' across T stage of BLCA. Epigenotype clusters are clustered by chromatin accessibility on MIBC DMR. Cluster8/9/10/11/12/13/14 are basal cancer and Cluster5/6/7 are TPCS. (B) Flow map from epigenotype (left) to copy number variance (CNV, middle) and RNA phenotype in integrated scATAC data. Cells are coloured by their RNA phenotype (bottom). (C) Lower: heatmap of single cell epigenotype ( $\mathrm{x}$, columns) matching to 
987 988

single cell RNA cluster (y, rows). RNA TPCS cells matched only to epigenotype cluster 5/6/7, which are labelled as TPCS-associated epigenotype. Top: entropy in each epigenotype cluster shows that TPCS-associated epigenotypes match significantly more RNA phenotype than nonTPCS-associated epigenotypes. (D) Cytotrace-inferred differentiation potential (high: 1, low: 0) of each epithelial cell types. Differences between groups were measured by adjusted Wilcox test $(* * *: \mathrm{P}<0.001, * *: \mathrm{P}<0.01, *: \mathrm{P}<0.05)$. Compared to TPCS, FPC and the urothelial progenitor cells are stem-like, and basal and luminal cancer cells are more differentiated.

\section{Figure 7. TPCS dynamically regulates neoantigen expression to evade immunoediting.}

(A) The prevalence of $\mathrm{T}$ cells with(+)/without(-) clonal expansion across BLCA T stages. 100\% advanced $\mathrm{T}$ stage BLCA patients have clonally expanded $\mathrm{T}$ cells in tumour tissue. (B) TCR clonotype diversity (Shannon entropy) across clinical stages shows a general trend of increase in clonotype diversity in the advanced clinical stage. (C-D) Clonal expansion (C) and gene expression (D) of each class of CD4 T helper cells. Colour in (C): red, singlet; orange, localexpanded ( $>2$ similar TCR found only in bladder tissue); and blue, global-expanded ( $>2$ similar TCR found in multiple tissue samples from the same donor). Colour in (D): blue, global-expanded; pink and orange, local-expanded. (E) PD-L1 cell-cell signalling with all bladder tissue cells revealing that $\operatorname{tr} \mathrm{T}_{\text {reg }}$ is the main, if not the only one, contributor of PD-L1 ligand for other tumourreactive T cells. (F-G) The prevalence of trTh1 (F) and $\operatorname{tr} \mathrm{T}_{\text {reg }}(\mathrm{G})$ in cancer and adjacent tissues. (H-I) Clonal expansion (H) and gene expression (I) of each class of CD8 cytotoxic T cells. Colour annotation is the same with (C-D). (J-L) The prevalence of $\operatorname{tr} \operatorname{T}_{\text {eff IFNGlo }}(\mathrm{J})$, $\operatorname{trTeff}$ IFNGhi $(\mathrm{K})$, and trT eff $_{\text {CXCR6 }}$ (L) in cancer and adjacent tissues. (M) Frequency of single cancer and TPCS cells expressing mutation-carrying transcripts in each donor. (N) Normalized per-cell mutation-carrying 
1010 transcript expression level, separated by clinical stage. (O-P) TPCS metagene activity (O) and

1011 survival probability (P) in IMVigor210 cohort. Samples are classified as immune desert, excluded,

1012 or inflamed as in the original paper.

1013

1014 Figure 8. TPCS dynamically shifts cell cycle state to escape from chemotherapy.

1015 (A) Epigenotype-phenotype matching entropy for each epigenotype cluster. Cells are separated by 1016 their donor into treatment-naïve and post-chemotherapy groups. TPCS has high epigenotype 1017 plasticity regardless of chemotherapy induction. (B) Epigenotype prevalence in treatment-naïve 1018 and post-chemotherapy donors, showing that TPCS epigenotype is increased after chemotherapy 1019 treatment. (C) Epigenotype prevalence in stage 2 patients. (D) Epigenotype prevalence in stage 4 1020 patients. (E) Cell cycle phase of basal, luminal-like, FPC and TPCS cancer cells in treatment-naïve 1021 and post-chemotherapy samples. TPCS shows lower proliferating potential in the treatment-naïve 1022 stage than FPC, but is the only cell type with elevated proliferating potential post-chemotherapy. 1023 (F) Survival probability of patients with high and low TPCS metagene activity in TCGA bladder 1024 cancer patient cohort. 
A
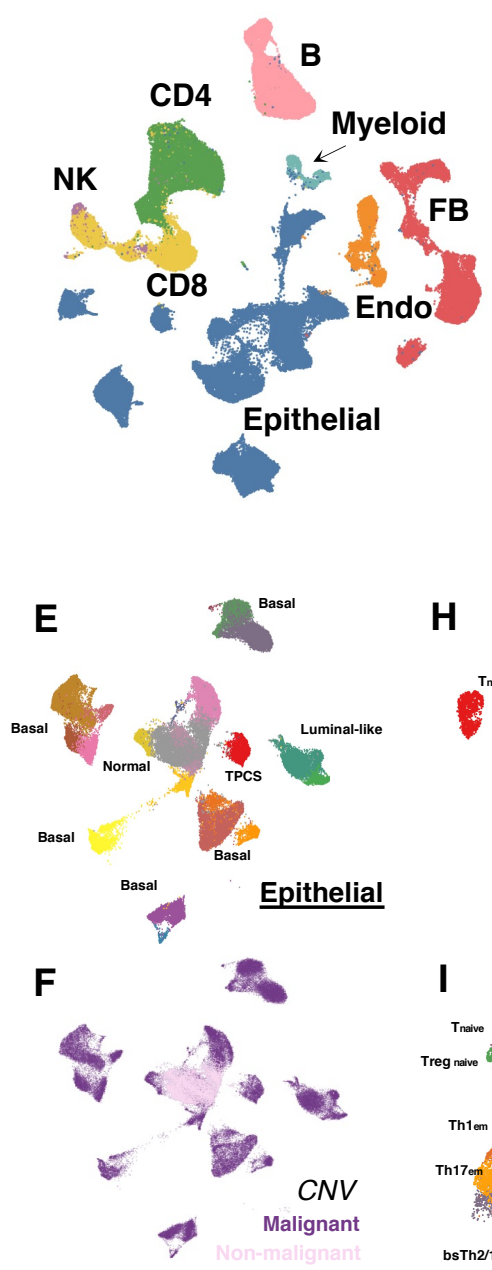

G

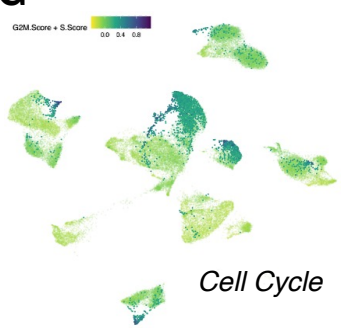

B

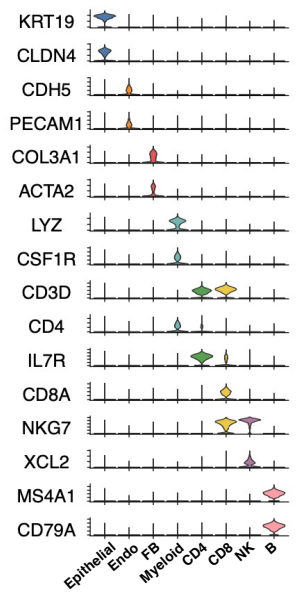

H
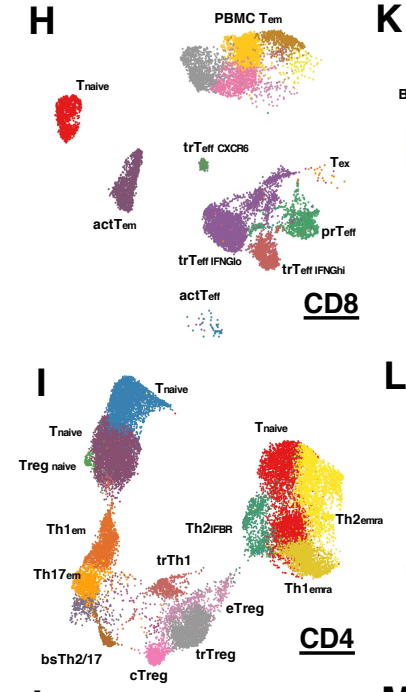

J

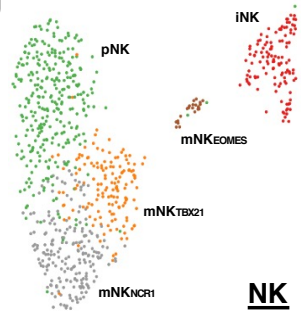

C

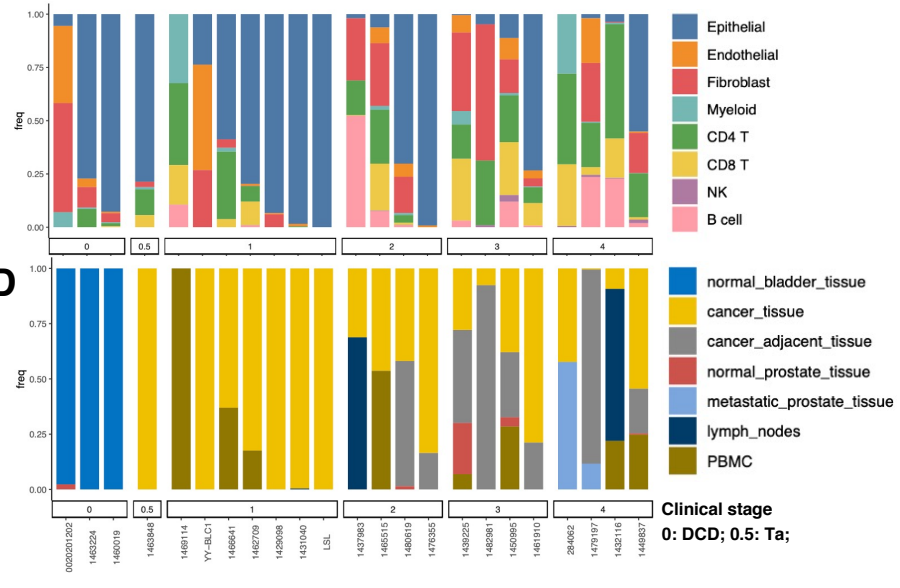

K
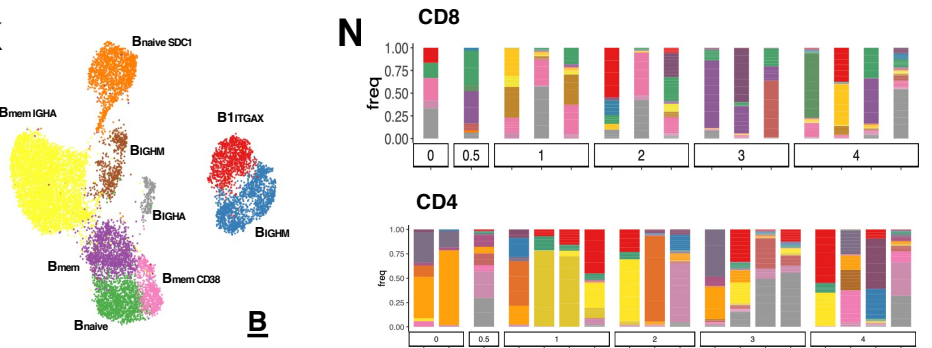

CD4

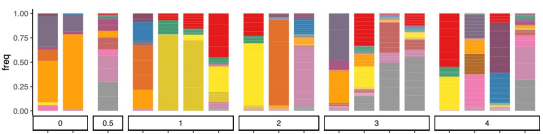

NK

NK

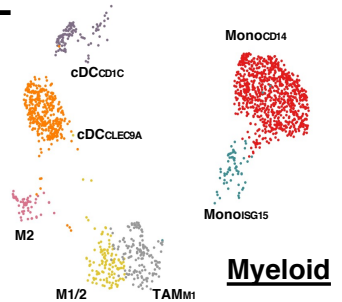

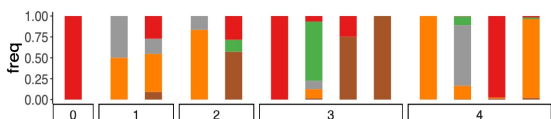

B

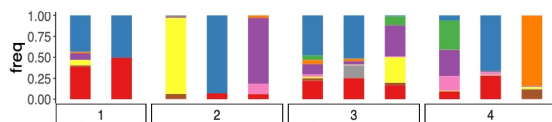

Myeloid

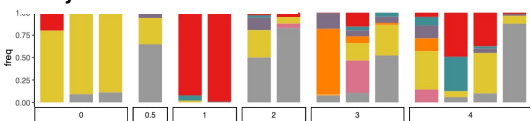

Fibroblast

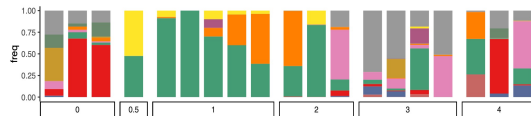

A note of cell type and markers in supplementary material

Figure 1. Single cell landscape of BLCA. 
A

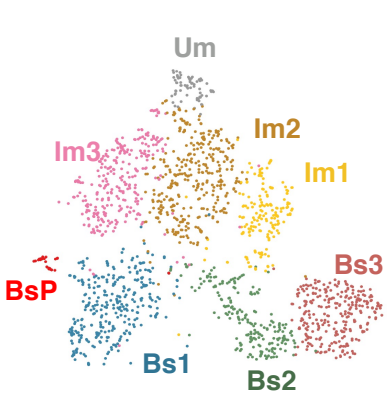

B

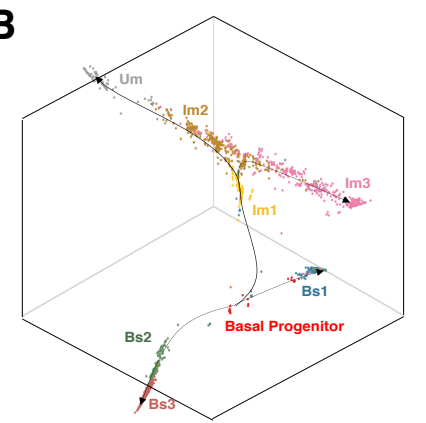

C

D
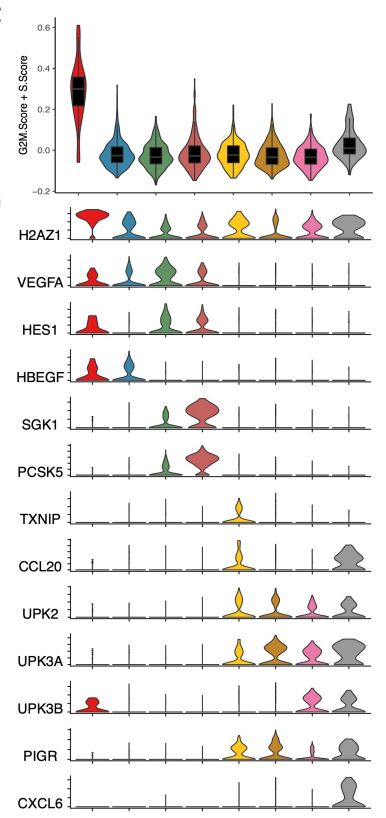

$\mathbf{E}$

UPK

G

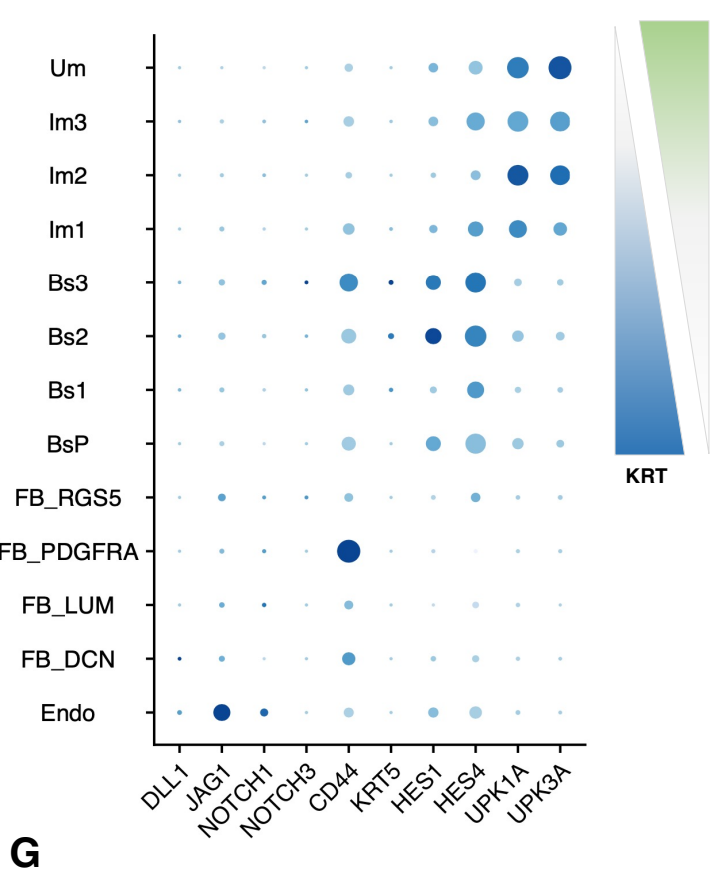

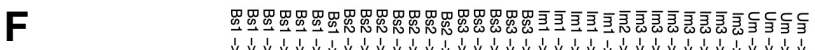

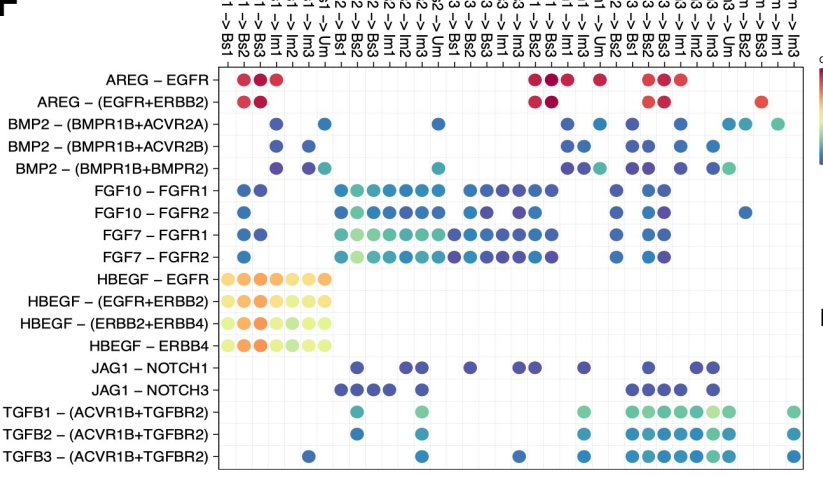

Um
Umbrella
Intermediate
Im1//m2/Im3
Basal
BsP/Bs1/Bs2/Bs3

Fibroblast JAG1+/DLL1+

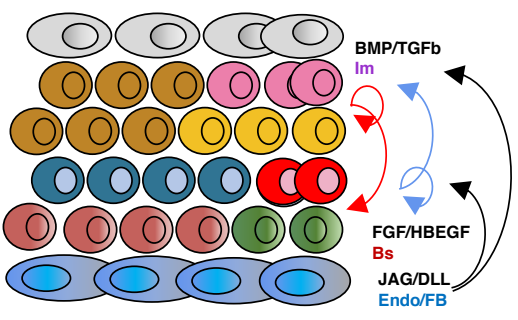

Figure 2. Bladder urothelium cell fate determination by mutual signaling. 


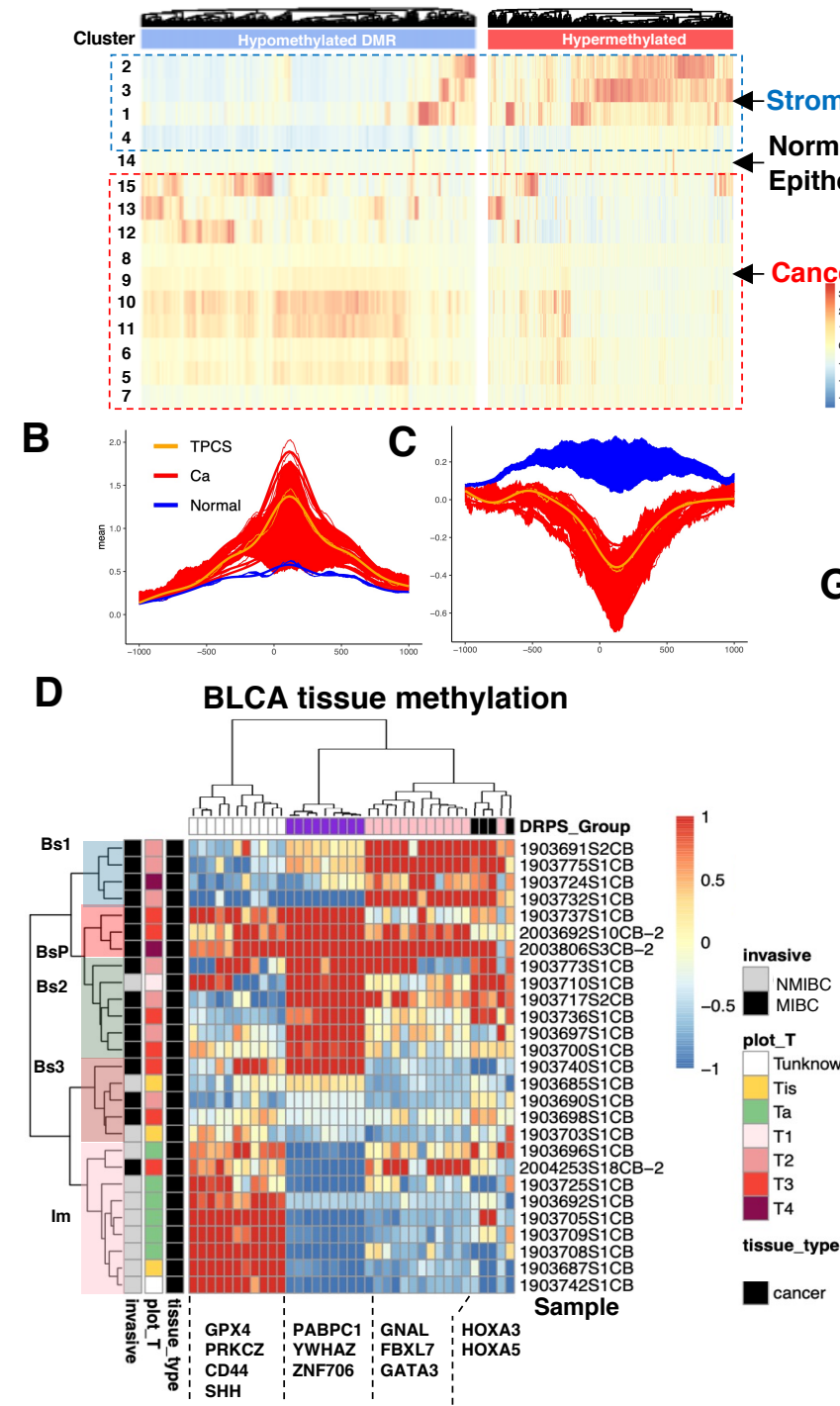

E Normal bladder scRNA gene expression

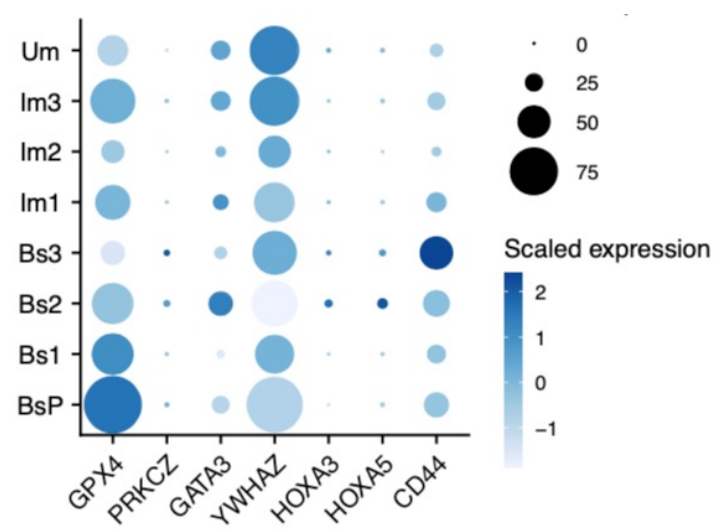

G

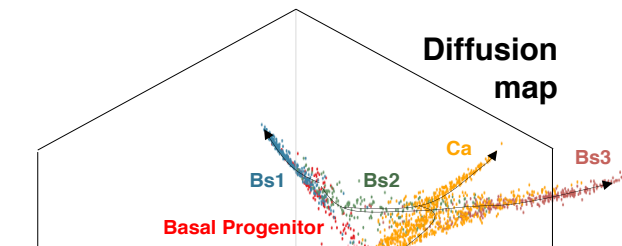

H Bs3

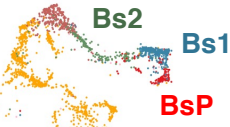
TPCS

Basal Progenitor
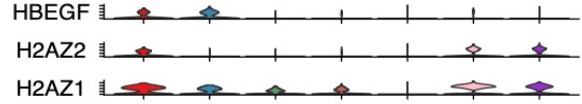

TM4SF1

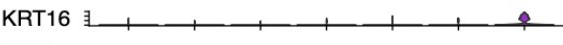

KRT14 年,

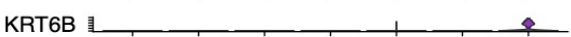

KRT6A 责, 上, , , , ,

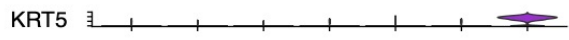

CD44 1: \&

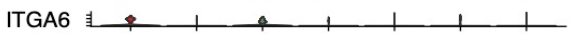

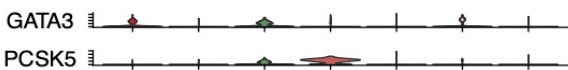

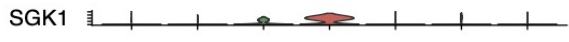

VEGFA I 1 \& \& \& \& \& 1

HES4 $1+4,111$

KRT17 HES1

KRT7 1

KRT8

Figure 3. Origin of muscle-invasive and TM4SF1-positive bladder cancer cells in urothelium. 
A scRNA Cell-cell signalling

BMP2 - (BMPR1B+ACVR2A)
BMP2 - (BMPR1B+ACVR2B)-

NMF16: SHH NFIB GLI1 MEF2C meth $_{\text {m }}$

D

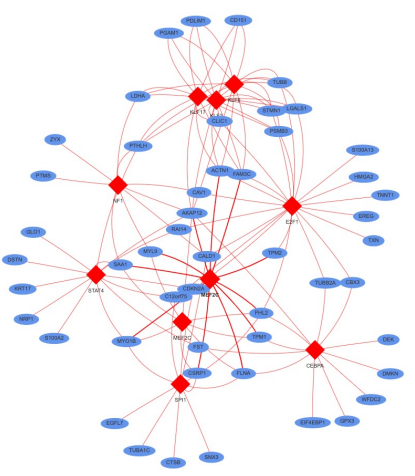

E

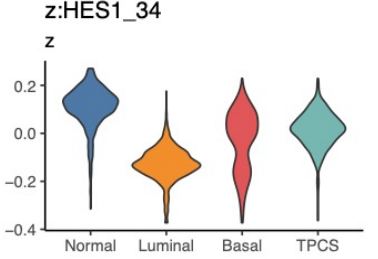

z:HES4_95

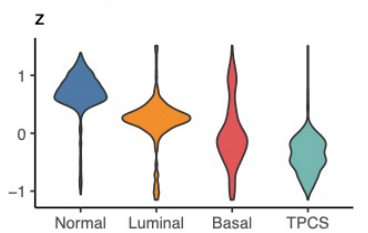

z:SMAD4_739

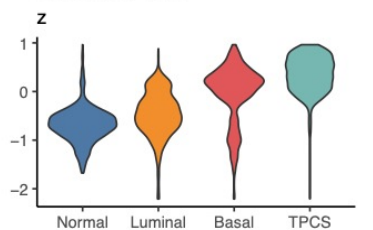

B

ScRNA expression

C ScRNA expression

NMF_6
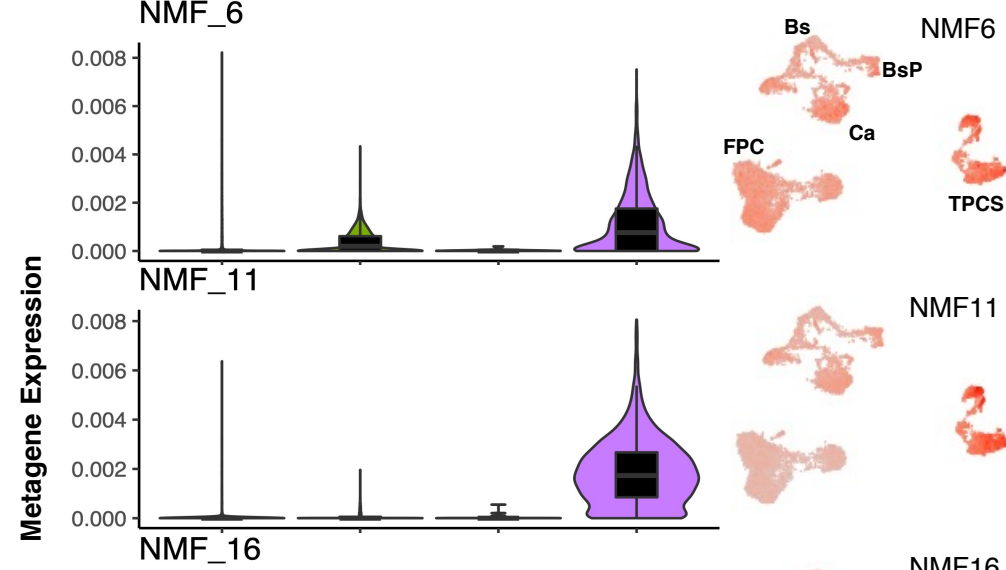

NMF11

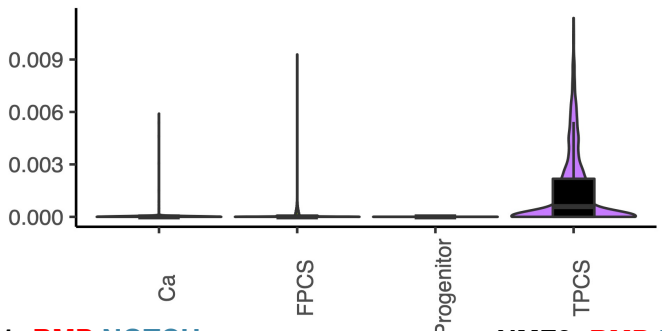

NMF16

NMF11: BMP NOTCH

KLF12 JDP2 SMAD4 ASCL2

NMF6: BMP NOTCH

SMAD5 ASCL2 HDAC6

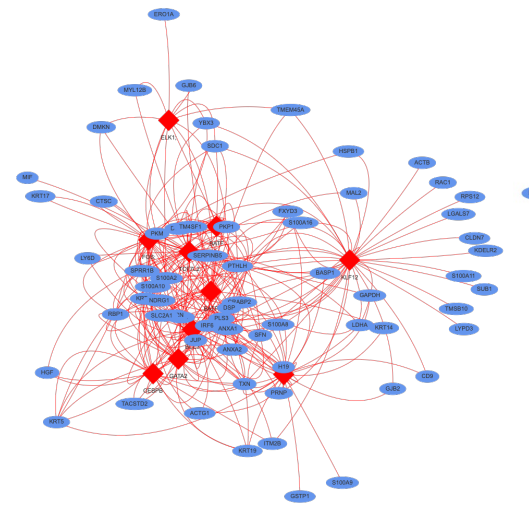

z:ASCL1_843

HES

Exp

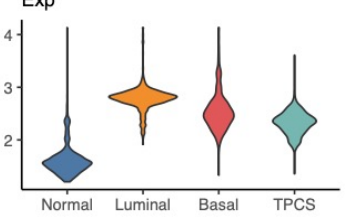

HES4

Exp

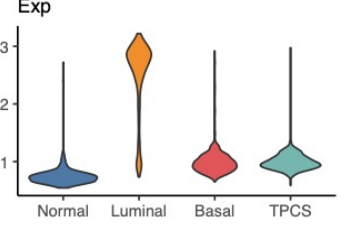

SMAD4

Exp

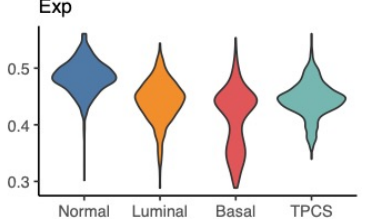

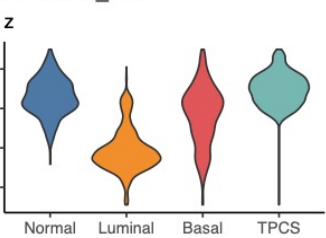

z:ASCL2_89

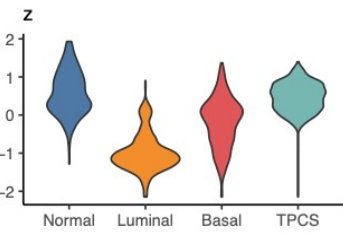

z:FOXA1_357

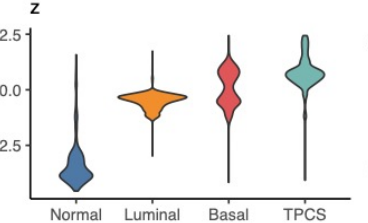

-

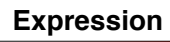

Low High

sCATAC TF binding activity measurement

Figure 4. Extrinsic and intrinsic determinants in TPCS transformation. 


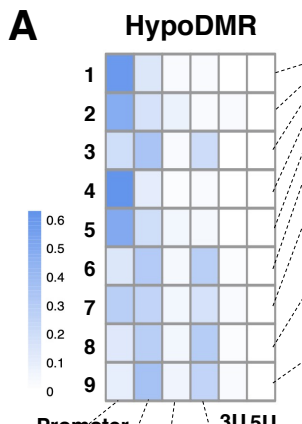

Promóter 3U5U Intron Exon Distal

B

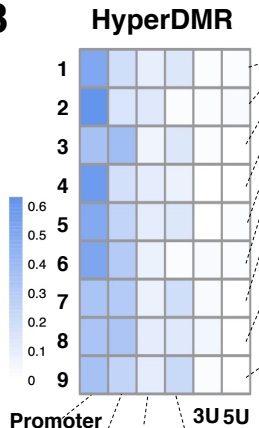

Promóter

$3 U 5 U$ Intron Exon Distal

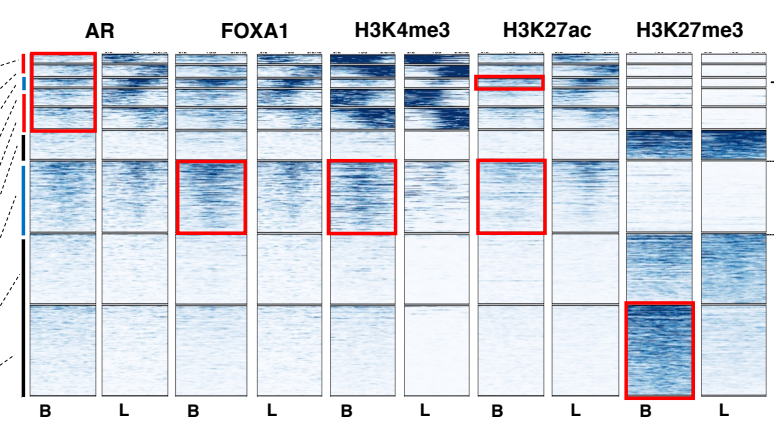

Promoter Enhancer Repressor

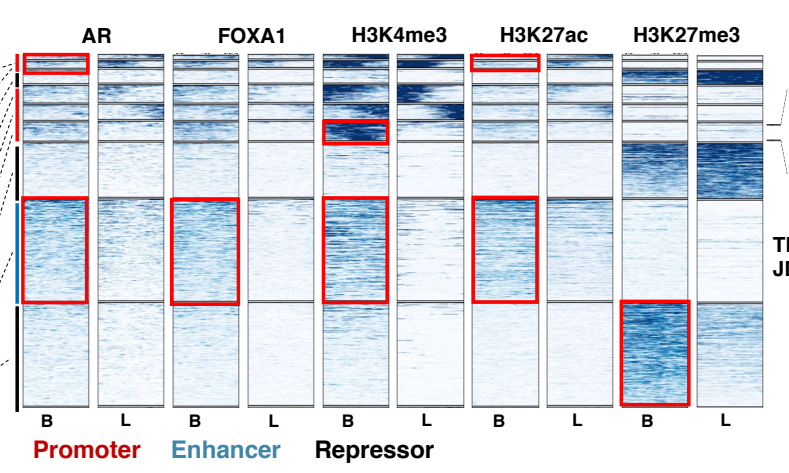

C SCATAC on hypoC3

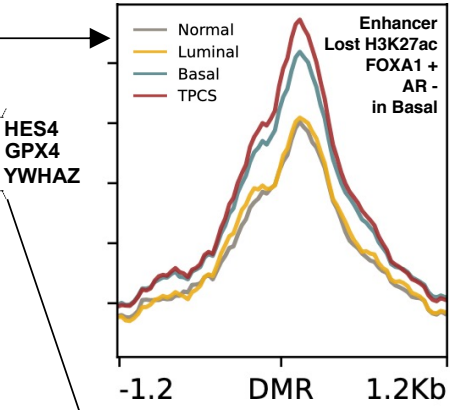

scATAC on hypoC7

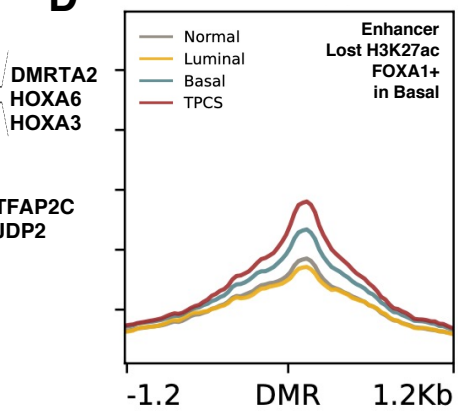

E

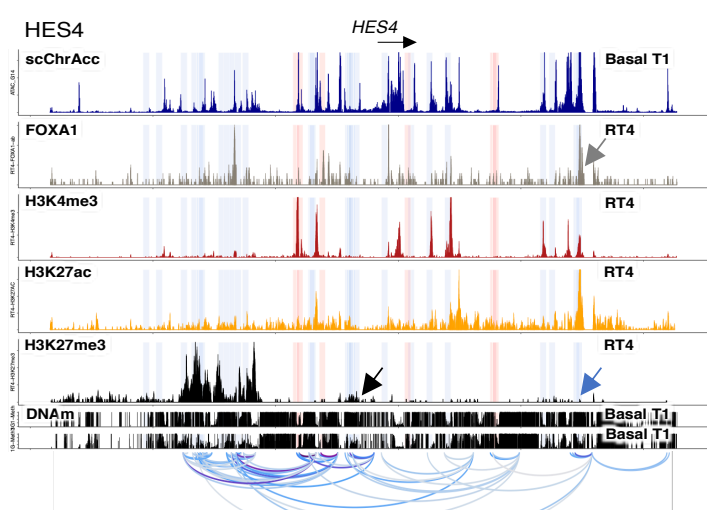

G

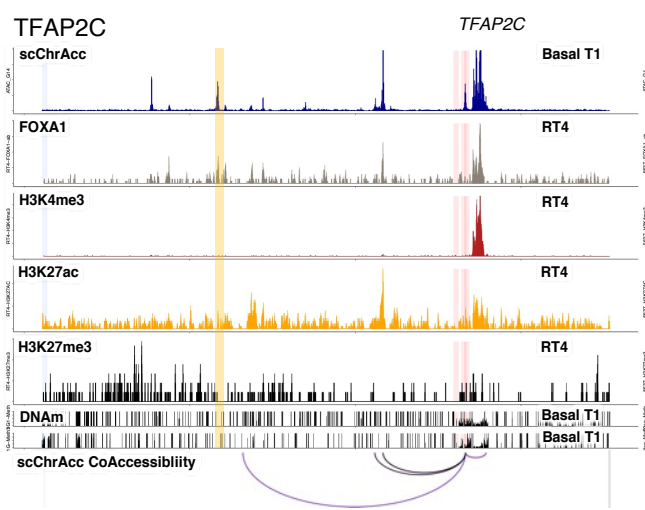

$\mathbf{F}$

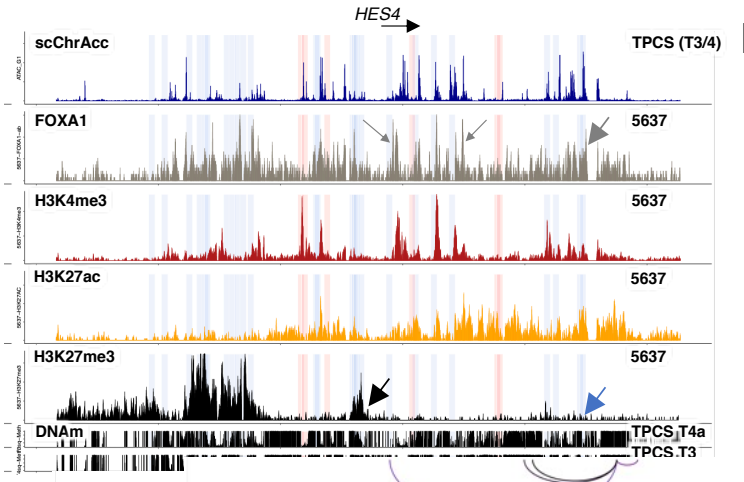

H

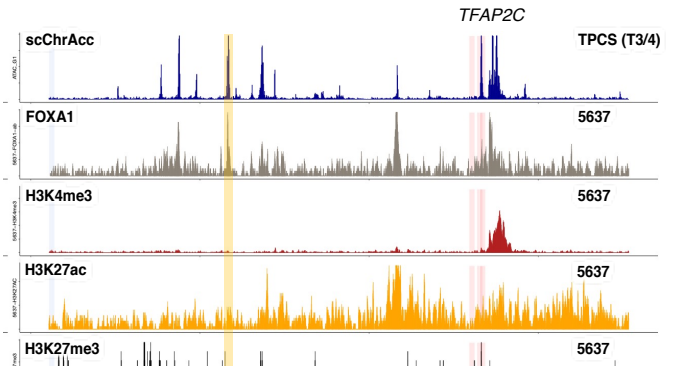

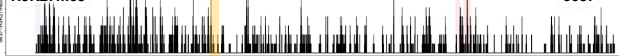

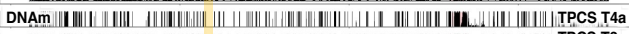

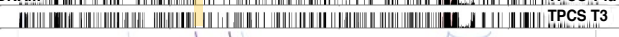

Figure 5. FOXA1 and AR binding reprogrammed enhancers epigenetically in TPCS. 
A

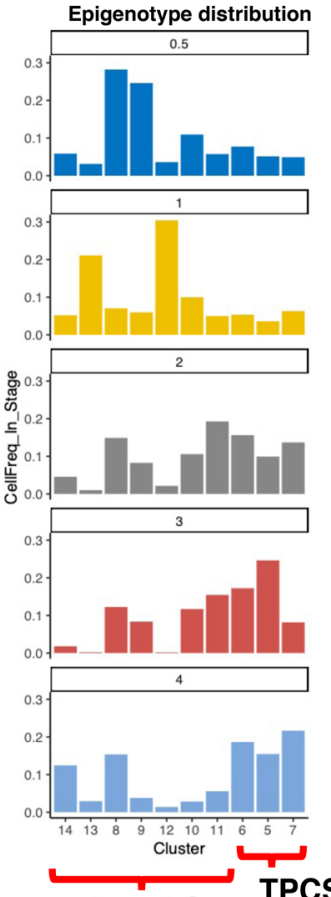

Basal Ca
B

Epigenotype-phenotype match

Epigenotype CNV RNA Phenotype

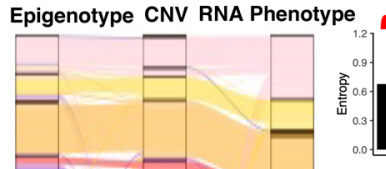

TPCS Basal Ca
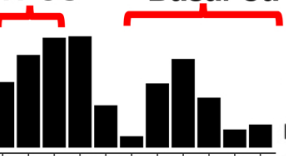

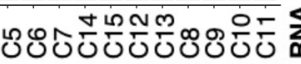

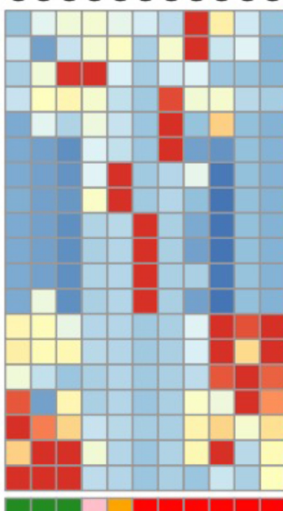

DMR All

RNA

ChrAcc ChrAcc

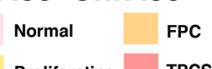

Proliferating TPCS
-

$\square$ FPC Luminal $\square \mathrm{Ca}$

\section{D}

Cell differentiation potential assessment

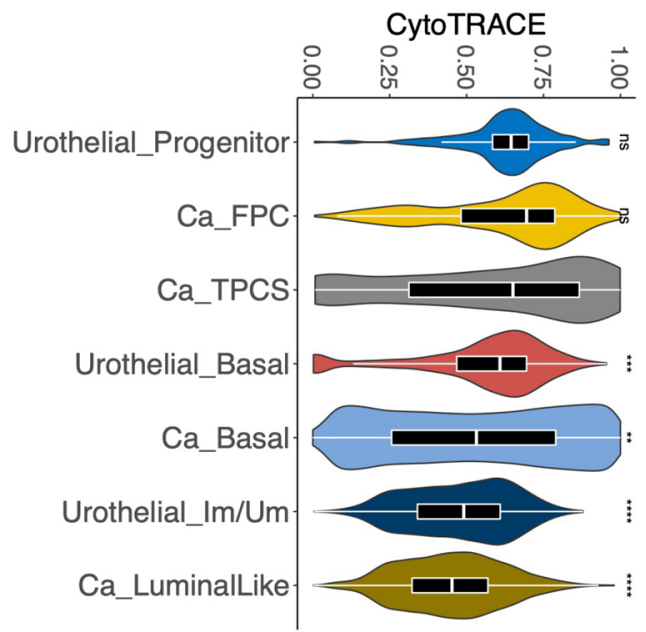

(Celi $)$ RNA type

Epigenotype

Epigenotype

Figure 6. Epigenotype of TPCS is phenotypically plastic. 
A

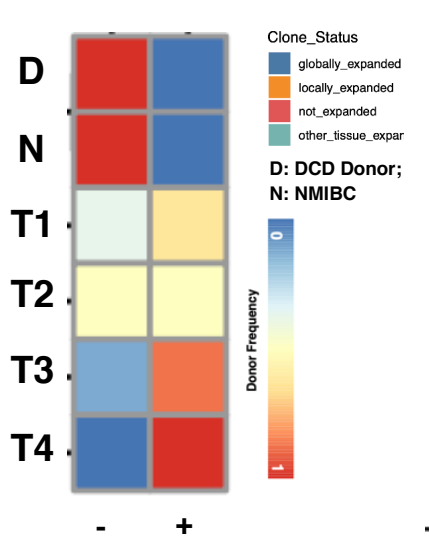

C

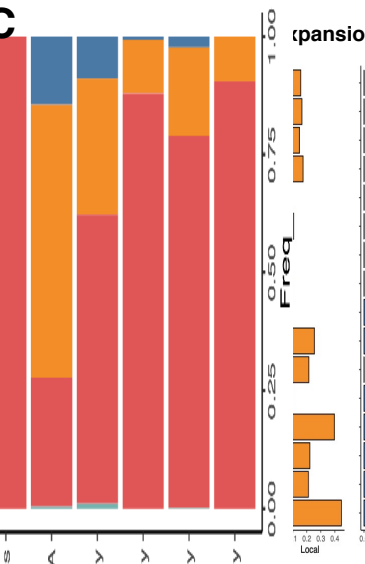

, $\mathbf{H}$
D

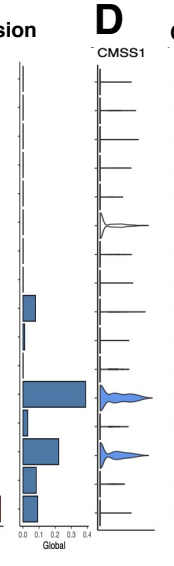

D4 gene expression
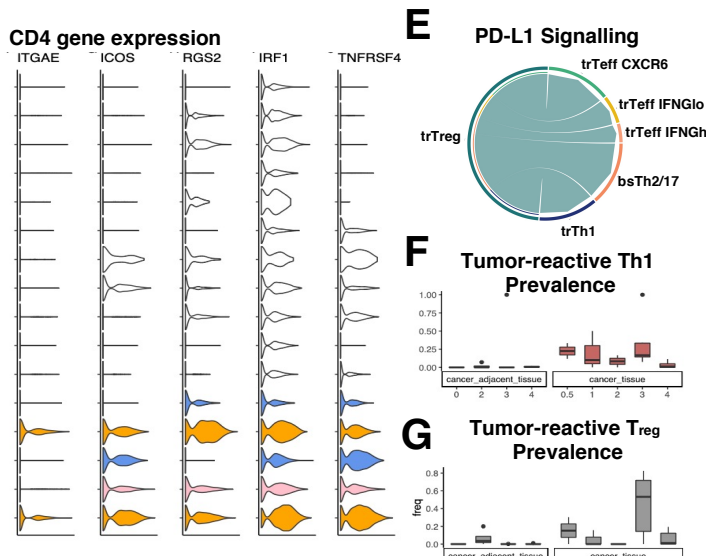

F Tumor-reactive Th1

B Expanded Clone

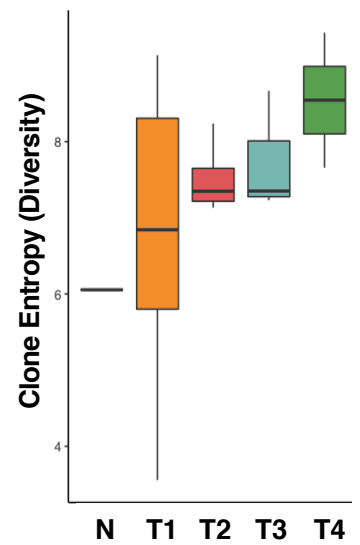

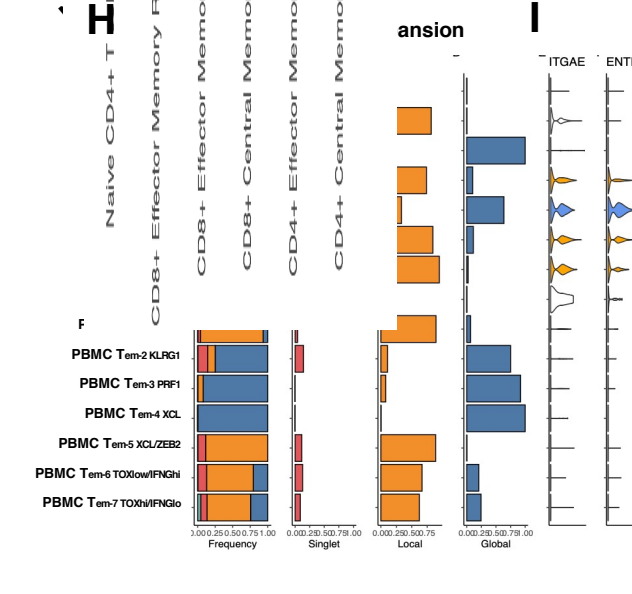

CD8 gene expression Prevalence.

J Tumor-reactive Tcyt Prevalence

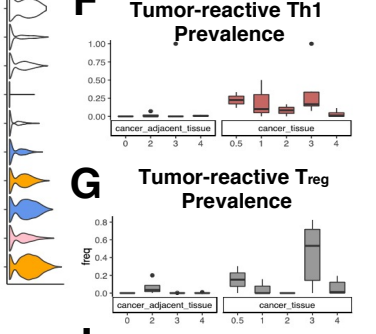

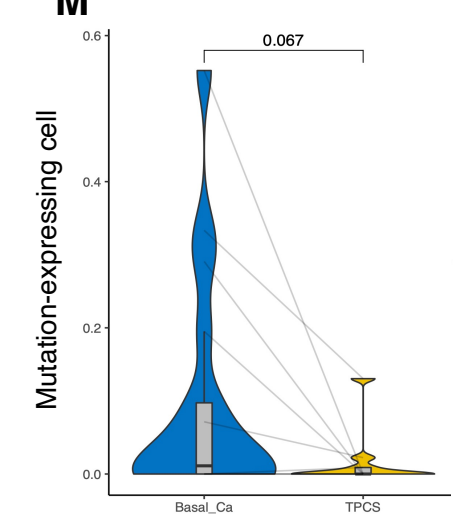
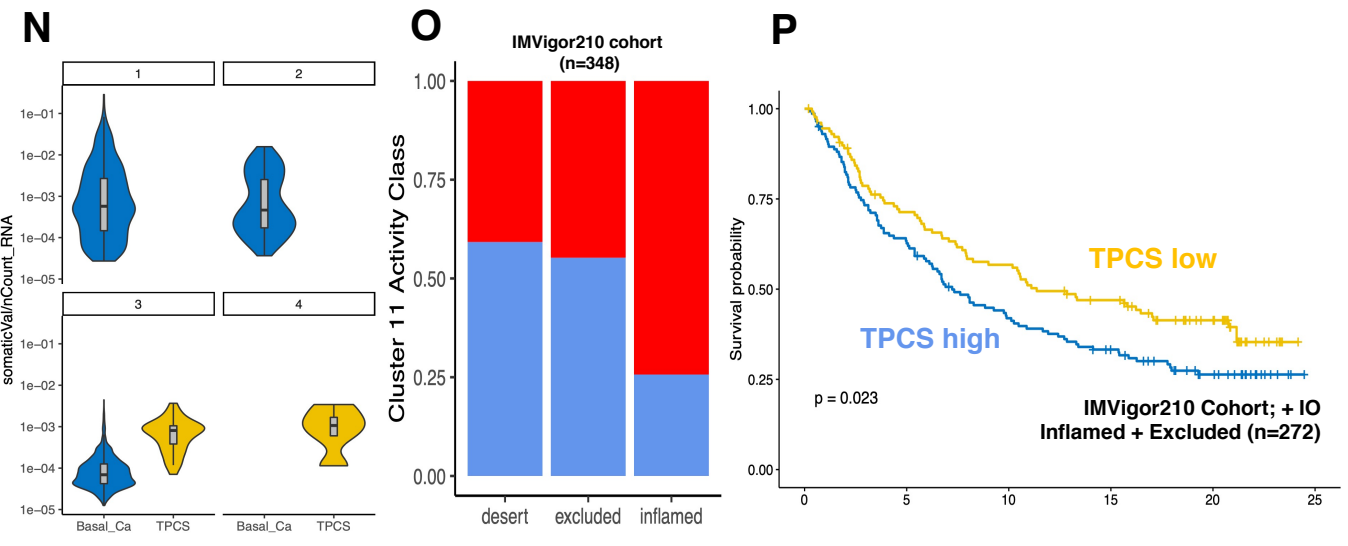

Figure 7. TPCS dynamically regulates neoantigen expression to evade immunoediting. 


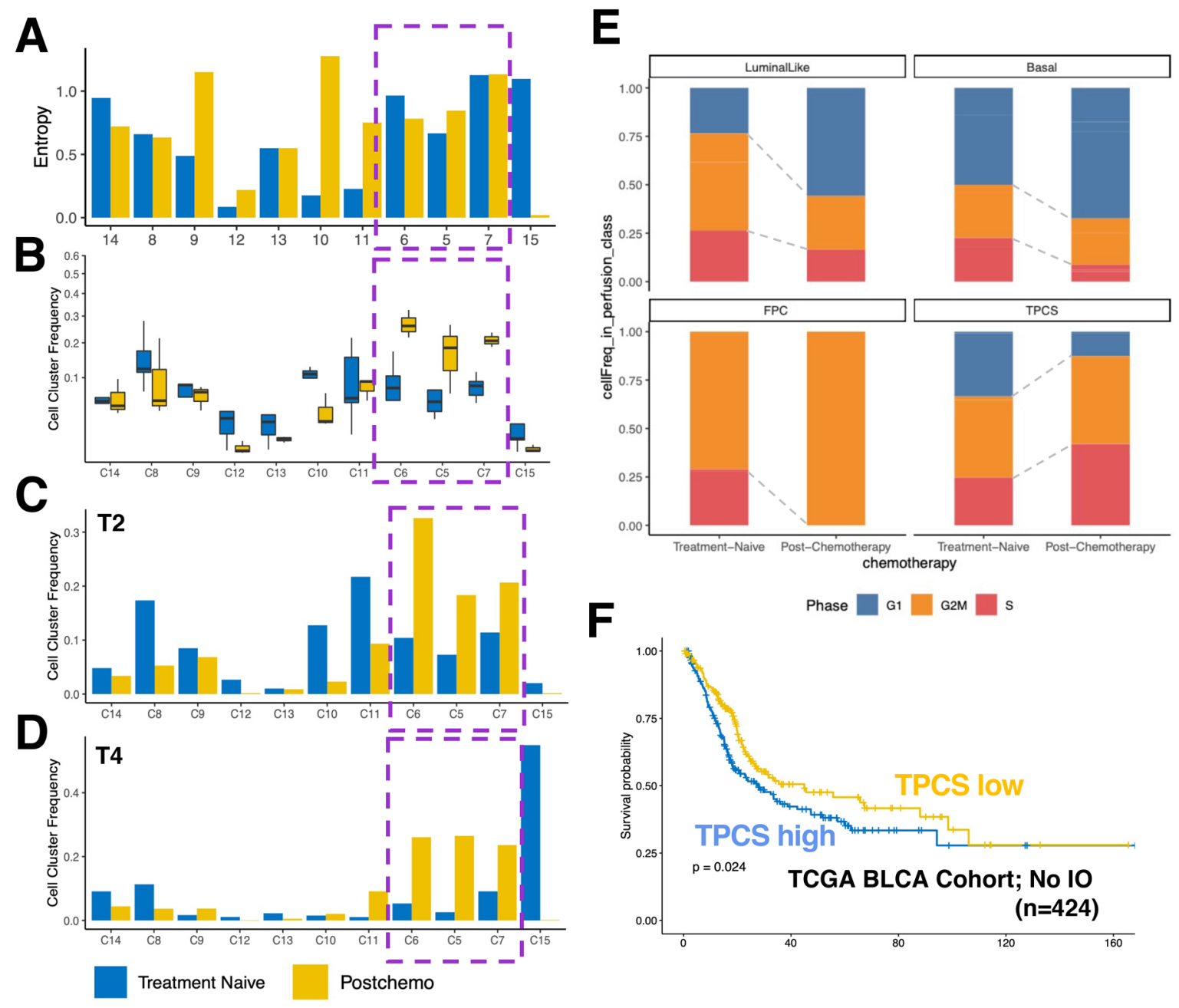

Figure 8. TPCS dynamically shifts cell cycle state to resist chemotherapy. 